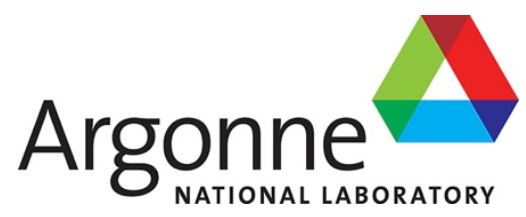

\title{
Progress Report on SAM Reduced-Order Model Development for Thermal Stratification and Mixing during Reactor Transients
}

Nuclear Engineering Division 


\begin{abstract}
About Argonne National Laboratory
Argonne is a U.S. Department of Energy laboratory managed by UChicago Argonne, LLC under contract DE-AC02-06CH11357. The Laboratory's main facility is outside Chicago,

at 9700 South Cass Avenue, Argonne, Illinois 60439. For information about Argonne

and its pioneering science and technology programs, see www.anl.gov.
\end{abstract}

\title{
DOCUMENT AVAILABILITY
}

Online Access: U.S. Department of Energy (DOE) reports produced after 1991 and a growing number of pre-1991 documents are available free via DOE's SciTech Connect (http://www.osti.gov/scitech/)

Reports not in digital format may be purchased by the public from the National Technical Information Service (NTIS):

U.S. Department of Commerce

National Technical Information Service

5301 Shawnee Rd

Alexandra, VA 22312

www.ntis.gov

Phone: (800) 553-NTIS (6847) or (703) 605-6000

Fax: (703) 605-6900

Email: orders@ntis.gov

Reports not in digital format are available to DOE and DOE contractors from the Office of Scientific and Technical Information (OSTI):

U.S. Department of Energy

Office of Scientific and Technical Information

P.O. Box 62

Oak Ridge, TN 37831-0062

www.osti.gov

Phone: (865) 576-8401

Fax: (865) 576-5728

\section{Disclaimer}

This report was prepared as an account of work sponsored by an agency of the United States Government. Neither the United States Government nor any agency thereof, nor UChicago Argonne, LLC, nor any of their employees or officers, makes any warranty, express or implied, or assumes any legal liability or responsibility for the accuracy, completeness, or usefulness of any information, apparatus, product, or process disclosed, or represents that its use would not infringe privately owned rights. Reference herein to any specific commercial product, process, or service by trade name, trademark, manufacturer, or otherwise, does not necessarily constitute or imply its endorsement, recommendation, or favoring by the United States Government or any agency thereof. The views and opinions of document authors expressed herein do not necessarily state or reflect those of the United States Government or any agency thereof, Argonne National Laboratory, or UChicago Argonne, LLC 
ANL/NE-17/29

\section{Progress Report on SAM Reduced-Order Model Development for Thermal Stratification and Mixing during Reactor Transients}

prepared by

R. $\mathrm{Hu}$

Nuclear Engineering Division, Argonne National Laboratory

September 2017 



\section{EXECUTIVE SUMMARY}

Mixing, thermal-stratification, and mass transport phenomena in large pools or enclosures play major roles for the safety of reactor systems. Depending on the fidelity requirement and computational resources, various modeling methods, from the 0 -D perfect mixing model to 3D Computational Fluid Dynamics (CFD) models, are available. Each is associated with its own advantages and shortcomings. It is very desirable to develop an advanced and efficient thermal mixing and stratification modeling capability embedded in a modern system analysis code to improve the accuracy of reactor safety analyses and to reduce modeling uncertainties.

An advanced system analysis tool, SAM, is being developed at Argonne National Laboratory for advanced non-LWR reactor safety analysis, under the support of U.S. Department of Energy (DOE) Nuclear Energy Advanced Modeling and Simulation (NEAMS) program. It aims to provide fast-running, modest-fidelity, whole-plant transient analyses capabilities, which are essential for fast turnaround design scoping and engineering analyses of advanced reactor concepts. While SAM is being developed as a system-level modeling and simulation tool, advanced modeling techniques are under development to tackle the issue of thermal mixing and stratification modeling in large enclosures of reactor systems during transients.

This report documents the initial progress on the reduced-order flow model developments in SAM for thermal stratification and mixing modeling. Two different modeling approaches are pursued. The first one is based on one-dimensional fluid equations with additional terms accounting for the thermal mixing from both flow circulations and turbulent mixing. The second approach is based on three-dimensional coarse-grid CFD approach, in which the full three-dimensional fluid conservation equations are modeled with closure models to account for the effects of turbulence.

The SAM 1-D modeling approach is based on the concept that the large enclosure can be divided into a number of sub-regions, separated by horizontal interfaces. The inter-volume energy exchange can be modeled by both advection and flow mixing. A simplified version of the 1-D modeling approach is to model the large enclosure with multiple 0-D volumes. The feasibility of the multiple 0 -D volume modeling approach was tested using the EBR-II lossof-heat-sink test BOP-302R. Focused on the thermal-hydraulics responses of the system throughout the transients in which the reactor power history was specified in the input model, very good agreement was found among the code simulations and the test results. The highand low-pressure inlet plena temperatures from the SAM simulation agreed very well with the test for both the initial heat up rates and the later pseudo-equilibrium states. It is thus demonstrated that it is feasible to account for the effects of thermal stratification in the cold pool by a simple two-volume cold pool model. The simulation results were highly dependent on the assumption of the mixing flow (fixed at $50 \%$ of normal core flow rate to best match the experimental results) between the upper and lower cold pool. Therefore, CFD simulations of the EBR-II cold pool during BOP-302R test was performed, from which the mixing flow between the two volume can be derived. It was found that the mixing flow rate was not constant and the magnitude varied significantly throughout the transient. This indicate that the assumption on the mixing flow rate used in stand-alone SAM simulation is not correct, even 
though the simulation results matched well with the experimental results. Further study is needed to develop correlations between the mixing flow rates at horizontal cross planes and the lumped sub-volume parameters.

A reduced-order three-dimensional module is also under development to model the multidimensional flow and thermal mixing and stratification in large enclosures of reactor systems. For computationally efficient modeling capabilities, the key modeling approaches include: 1) Solving the full 3-D fluid conservation equations; 2) Using only coarse computational meshes; 3) No turbulence modeling; 4) Developing closure models to account for the effects of turbulence and the use of coarse mesh in momentum and energy transport. The framework of a 3-D FEM flow model has been developed and implemented in SAM. To prevent the potential numerical instability issues, the SUPG and PSPG formulations have been implemented. Several verification and validation tests were performed, including lid-driven cavity flow, natural convection inside a cavity, and laminar flow in a channel of parallel plates. Based on the comparisons with the analytical solutions and experimental results, it is demonstrated that the developed 3-D fluid model can perform very well for a range of laminar flow problems. This 3-D flow model is based on solving the primitive variables in the conservative form of the governing equations for incompressible but thermally expandable flows. Combined with the use of the JFNK solution method and high-order discretization schemes, this flow model has great potential for both efficient and accurate multi-dimensional flow simulations. Continued developments will be focused on closure model developments to capture the effects of turbulence and the use of coarse mesh in momentum and energy transport, and on additional code verifications and validations close to reactor conditions. 


\section{Table of Contents}

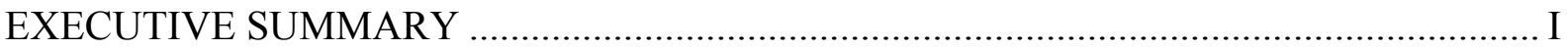

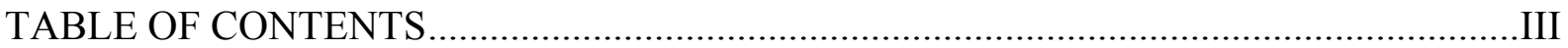

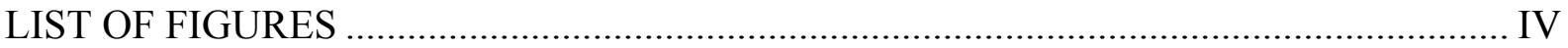

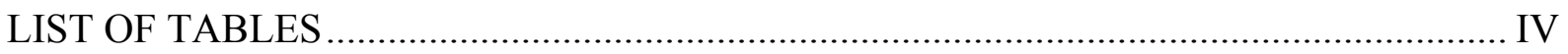

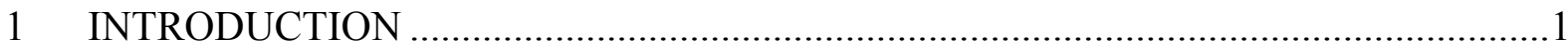

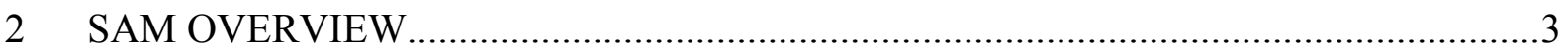

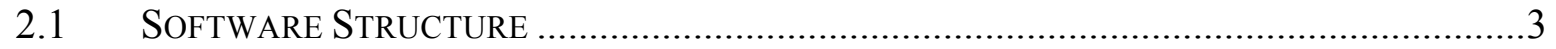

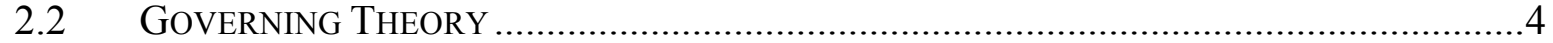

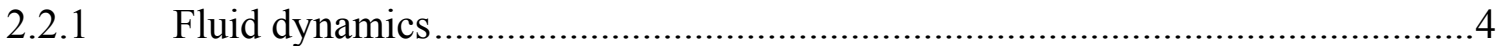

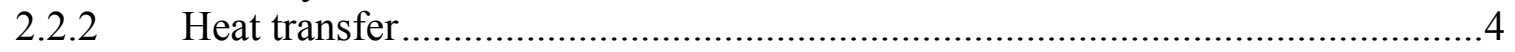

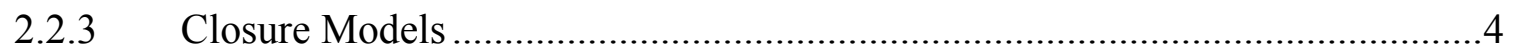

2.2.4 Numerical Methods...............................................................................

2.3 OVERVIEW OF CURRENT CAPABILITIES ………………...........................................

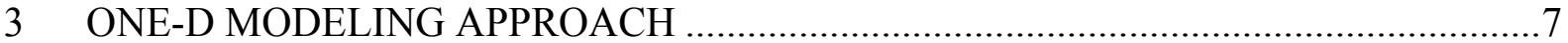

3.1 Multiple 0-D Volume MOdeling APPROACH .....................................................

3.2 DEMONSTRATION SIMULATION OF EBR-II TRANSIENT …….......................................

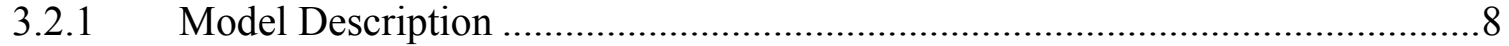

3.2.2 Simulation Results ...............................................................................12

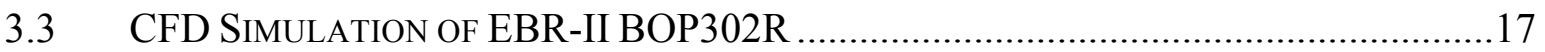

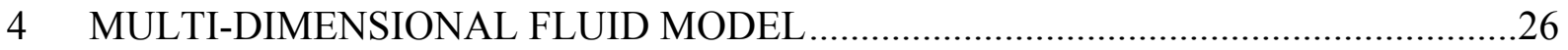

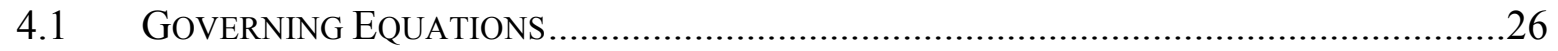

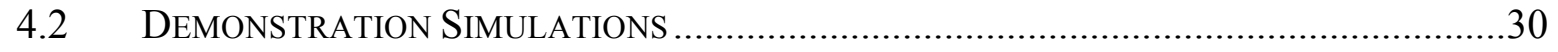

4.2.1 Lid-driven cavity flow ……………………….........................................30

4.2.2 Internal flow between parallel plates ..........................................................33

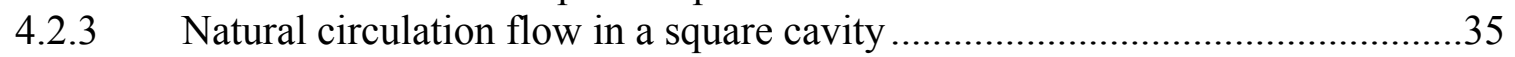

4.3 SUMMARY AND PATH FORWARD .........................................................................

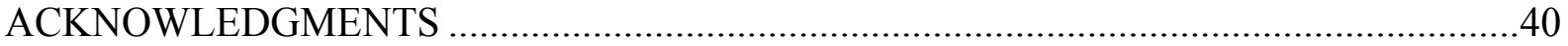

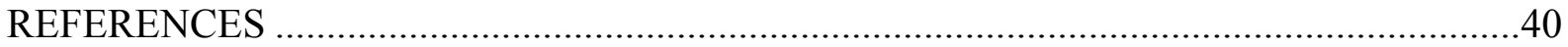




\section{LIST OF FIGURES}

Figure 1. SAM Code Structure 4

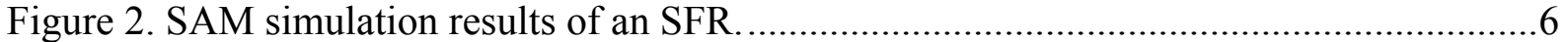

Figure 3. SAM Core Channel Model of EBR-II.................................................................

Figure 4. SAM Core Channel Model of EBR-II.................................................................. 11

Figure 5. EBR-II Primary Sodium System Model..................................................................... 12

Figure 6. Reactor power and IHX heat removal rate during BOP-302R test ............................14

Figure 7. SAM predictions of plena temperatures during BOP-302R test ...............................14

Figure 8. High-pressure inlet plenum temperature during BOP-302R test ..............................15

Figure 9. Low-pressure inlet plenum temperature during BOP-302R test ................................15

Figure 10. Z-Pipe inlet temperature during BOP-302R test ................................................. 16

Figure 11. IHX primary inlet temperature during BOP-302R test ..........................................16

Figure 12. Subassembly 6C4 outlet temperature during BOP-302R test .................................17

Figure 13. EBR-II geometries with STAR-CCM+CAD modeller .........................................19

Figure 14. IHX outlet temperatures during the BOP-302R test...............................................19

Figure 15. CFD prediction of the steady-state velocity distributions before BOP-302R

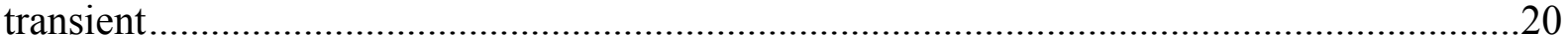

Figure 16. CFD prediction of the velocity distributions during BOP-302R transient ..............21

Figure 17. CFD prediction of the temperature distributions during BOP-302R transient........22

Figure 18. Mixing flow rates at various horizontal cross planes during the BOP-302R test ...24

Figure 19. Mixing flow rates and $R i$ at IHX bottom cross plane during the BOP-302R test ...25

Figure 20: Schematic of the lid-driven cavity flow problem...................................................

Figure 21: Results of the lid-driven cavity flow problem at various $R e$.................................32

Figure 22: Comparisons between SAM and experiment results for the lid-driven cavity flow

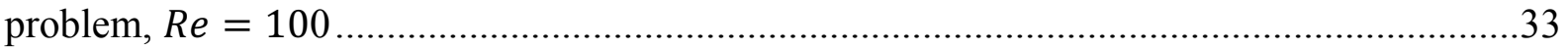

Figure 23: Schematic of flow in a channel of parallel plates...................................................33

Figure 24: Velocity distributions at different distances from flow inlet....................................34

Figure 25: Schematic of natural circulation flow problem in a square cavity ...........................35

Figure 26: Temperature distributions in the square cavity at various $R a$.................................36

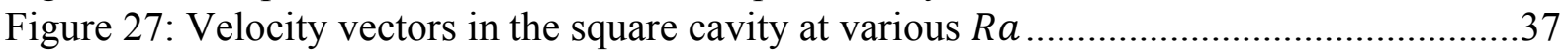

Figure 28: Temperature distributions in the square cavity at various $R a, y / L=0.5 \ldots \ldots \ldots \ldots \ldots . . .38$

Figure 29: Comparison between experiment and SAM predictions, $R a=1.89 \times 105, y / L=$

0.5 .38

\section{LIST OF TABLES}

Table 1. SAM lumped core channels 


\section{Introduction}

Mixing, thermal-stratification, and mass transport phenomena in large pools or enclosures play major roles for the safety of reactor systems. Such phenomena include the cold and hot pool mixing in pool-type Sodium-cooled Fast Reactors (SFR), reactor cavity cooling system behavior in High Temperature Gas-cooled Reactors (HTGR), passive containment cooling in advanced light-water reactors (such as AP1000), and thermal-stratification in BWR suppression pools. It is very important to accurately predict pool temperature and density distributions for both design optimizations and safety analyses of these reactor systems. However, the individual transport mechanisms governing mixing are characterized by time and length scales that can differ by orders of magnitude. Large volumes and complex interactions of different flow and thermal structures make the analysis of mixing in a large enclosure a very challenging task (intractable by analytic means and extremely demanding from a computational standpoint). Due to these reasons, experimental efforts [1-3] including large facilities like PANDA [3,4] have been continuously investigating these phenomena over the past three decades.

Depending on the fidelity requirement and computational resources, 0-D steady state models, 0-D lumped parameter based transient models, 1-D physical-based models, and 3-D Computational Fluid Dynamics (CFD) models are available. A good overview on major modeling methods of thermal mixing and stratification phenomena and their advantages and limits can be found in Reference [5].

Current major system analysis or severe accident analysis codes (such as SAS4A/SASSYS16], RELAP5[7], CATHRE[8], and MELCOR[9]) either have no models or only 0-D models for thermal mixing and stratification in large enclosures. The lack of general thermal mixing and stratification models in those codes severely limits their application and accuracy for safety analysis, especially for reactors relying on natural circulation for long-term cooling.

For example, the SAS4A/SASSYS-1 code developed by Argonne National Laboratory ("Argonne"), one of the major SFR system analysis codes, provides lumped-volume-based 0-D models that can only give very approximate results and can only handle simple cases with one mixing source. The models were derived according to simulant experiments for specific SFR upper plenum design configurations. Depending on the momentum and buoyancy of the outlet flow from the reactor core, well mixed case, two-zone with a negative buoyant jet case, two-zone with a positive buoyant jet case, and even more complex three-zone cases may form. The total jet entrainment, zone interface location, and average temperatures in each zone can be estimated by empirical correlations. Since the methods are based on scaled experimental data, using those models for SFR designs with different hot/cold pool configurations tends to result in large uncertainties.

Scaling analyses for prediction of thermal stratification and mixing in pools and in large interconnected enclosures were developed and applied by Peterson and coworkers at UC Berkeley [10,11]. A 1-D simulation code BMIX/BMIX++ was also developed to simulate stratification development in stably stratified conditions [12]. The ambient fluid volume is represented by 1-D transient partial differential equations, and substructures such as free or wall jets are modeled with 1-D integral models. This allows very large reductions in computational effort compared to 3-D CFD modeling. It was validated against a number of benchmark problems $[13,14]$. However, BMIX ++ cannot model the transition cases where the fully stratified 
ambient or well-mixed ambient assumptions break down. For a transient where initially stratified pool is gradually mixed, it cannot help to infer about the time scale for such mixing processes.

Reynolds-Averaged Navier-Stokes (RANS) based CFD approaches are less expensive than higher fidelity Large Eddy Simulations (LES), and has been applied in SFR upper plenum simulation with very detailed geometric modeling of major structures [15]. However, it is still computationally overwhelming for long-transient, complex-flow simulations in engineering applications, particularly when sensitivity/uncertainty analysis is needed for design optimizations and safety analyses. Multi-scale modeling approaches (achieved by coupling 1-D system and 3D CFD codes) has been tried to simulate large and complex domains by modeling the large volume with a CFD code and the rest of the system with a system code [16,17]. This methodology can provide detailed information only where needed while providing system level information in the rest of the domain. However, coupling different codes employing different solver routines and operating at different spatial and temporal scales remains a challenge. A notable fundamental problem is that a reliable and accurate coupling scheme is largely missing in the treatment of momentum and energy exchange at the boundary between the CFD model and the system model. It is very desirable to have an advanced and efficient thermal mixing and stratification modeling capability embedded in a system analysis code to improve the accuracy of reactor safety analyses and to reduce modeling uncertainties.

An advanced system analysis tool, SAM [18], is being developed at Argonne National Laboratory for advanced non-LWR reactor safety analysis. It aims to provide fast-running, modest-fidelity, whole-plant transient analyses capabilities, which are essential for fast turnaround design scoping and engineering analyses of advanced reactor concepts. While SAM is being developed as a system-level modeling and simulation tool [19,20], advanced modeling techniques including a reduced-order three-dimensional module are under development to tackle the issue of thermal mixing and stratification modeling in large enclosures of reactor systems during transients.

This report presents the initial progress on the reduced-order flow model developments in SAM for thermal stratification and mixing modeling. Two different modeling approaches are pursued. The first one is based on one-dimensional fluid models with additional terms accounting for the thermal mixing from both macroscopic flow circulations and microscopic turbulent mixing. The second approach is based on three-dimensional coarse-grid CFD approach, in which the full three-dimensional fluid conservation equations are modeled with closure models to account for the effects of turbulence. The technical basis of the two modeling approaches are discussed first, followed by some initial demonstration simulations and validation tests. The details of each modeling approach are presented in Chapter 3 and 4, respectively. 


\section{SAM Overview}

The System Analysis Module (SAM) [18] is an advanced system analysis tool being developed at Argonne National Laboratory under the support of U.S. Department of Energy (DOE) Nuclear Energy Advanced Modeling and Simulation (NEAMS) program. It aims to be a modern system analysis code, which takes advantages of the advancements in computing power, software design, numerical methods, and physical models over the past two decades. SAM focuses on modeling advanced reactor concepts such as SFRs (sodium fast reactors), LFRs (leadcooled fast reactors), and FHRs (fluoride-salt-cooled high temperature reactors) or MSRs (molten salt reactors). These advanced concepts are distinguished from light-water reactors in their use of single-phase, low-pressure, high-temperature, and low Prandtl number (sodium and lead) coolants. This simple yet fundamental change has significant impacts on core and plant design, the types of materials used, component design and operation, fuel behavior, and the significance of the fundamental physics in play during transient plant simulations.

SAM is aimed to solve the tightly-coupled physical phenomena including fission reaction, heat transfer, fluid dynamics, and thermal-mechanical response in reactor structures, systems and components in a fully-coupled fashion but with reduced-order modeling approaches to facilitate rapid turn-around for design and safety optimization studies. As a new code development, the initial effort focused on developing modeling and simulation capabilities of the heat transfer and single-phase fluid dynamics responses in reactor systems.

\subsection{Software Structure}

SAM is being developed as a system-level modeling and simulation tool with higher fidelity (compared to existing system analysis tools), and with well-defined and validated simulation capabilities for advanced reactor systems. It provides fast-running, modest-fidelity, whole-plant transient analyses capabilities. To fulfill the code development, SAM utilizes the object-oriented application framework MOOSE [21] and its underlying meshing and finite-element library libMesh [22] and linear and non-linear solvers PETSc [23], to leverage the available advanced software environments and numerical methods. The high-order spatial discretization schemes, fully implicit and high-order time integration schemes, and the advanced solution method (such as the Jacobian-free Newton-Krylov (JFNK) method [24]) are the key aspects in developing an accurate and computationally efficient model in SAM.

The software structure of SAM is illustrated in Figure 1. In addition to the fundamental physics modeling of the single-phase fluid flow and heat transfer, SAM incorporates advances in the closure models (such as convective heat transfer correlations) for reactor system analysis developed over the past several decades. A set of Components, which integrate the associated physics modeling in the component, have been developed for friendly user interactions. This component-based modeling strategy is similar to what is implemented in RELAP-7 [25], which is also a MOOSE-based system analysis tool (focused on LWR simulations). A flexible coupling interface has been developed in SAM so that multi-scale, multi-physics modeling capabilities can be achieved by integrating with other higher-fidelity or conventional simulation tools. 


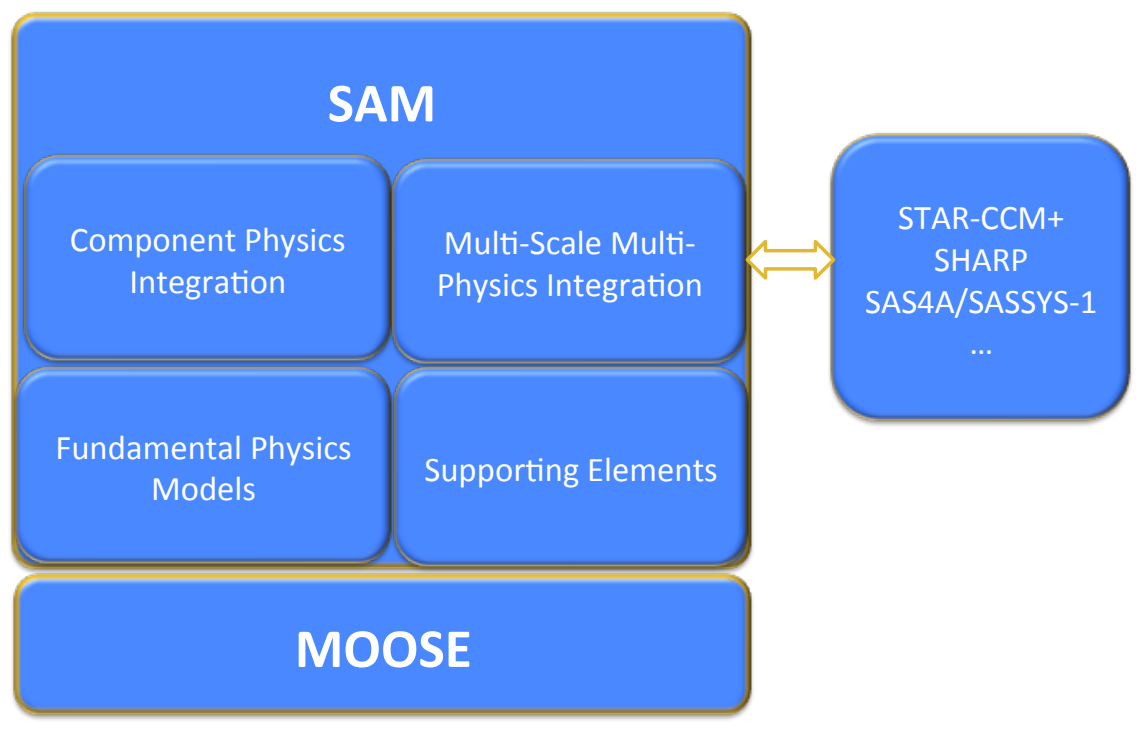

Figure 1. SAM Code Structure

\subsection{Governing Theory}

\subsubsection{Fluid dynamics}

Fluid dynamics is the main physical model of the SAM code. SAM employs a standard onedimensional transient model for single-phase incompressible but thermally expandable flow. The governing equations consist of the continuity equation, momentum equation, and energy equations. A three-dimensional module is also under development to model the multidimensional flow and thermal stratification in the upper plenum or the cold pool of an SFR. Additionally, a subchannel module will be developed for fuel assembly modeling.

\subsubsection{Heat transfer}

Heat structures model heat conduction inside solids and permit the modeling of heat transfer at interfaces between solid and fluid components. Heat structures are represented by onedimensional or two-dimensional heat conduction in Cartesian or cylindrical coordinates. Temperature-dependent thermal conductivities and volumetric heat capacities can be provided in tabular or functional form. Heat structures can be used to simulate the temperature distributions in solid components such as fuel pins or plates, heat exchanger tubes, and pipe and vessel walls, as well as to calculate the heat flux conditions for fluid components. Flexible conjugate heat transfer and thermal radiation modeling capabilities are also implemented in SAM.

\subsubsection{Closure Models}

The fluid equation of state (EOS) model is required to complete the governing flow equations, which are based on the primitive variable formulation; therefore, the dependency of fluid properties and their partial derivatives on the state variables (pressure and temperature) are implemented in the EOS model. Some fluid properties, such as sodium, air, salts like FLiBe and FLiNaK, have been implemented in SAM. Empirical correlations for friction factor and 
convective heat transfer coefficient are also required in SAM because of its one-dimension approximation of the flow field. The friction and heat transfer coefficients are dependent on flow geometries as well as operating conditions during the transient.

\subsubsection{Numerical Methods}

SAM is a finite-element-method based code. The "weak forms" of the governing equations are implemented in SAM. It uses the Jacobian-Free Newton Krylov (JFNK) solution method to solve the equation system. The JFNK method uses a multi-level approach, with outer Newton's iterations (nonlinear solver) and inner Krylov subspace methods (linear solver), in solving large nonlinear systems. The concept of 'Jacobian-free' is proposed, because deriving and assembling large Jacobian matrices could be difficult and expensive. The JFNK method has become an increasingly popular option for solving large nonlinear equation systems and multi-physics problems, as observed in a number of different disciplines [24]. One feature of JFNK is that all the unknowns are solved simultaneously in a fully coupled fashion. This solution scheme avoids the errors from operator splitting and is especially suitable for conjugate heat transfer problems in which heat conduction in a solid is tightly coupled with fluid flow.

\subsection{Overview of Current Capabilities}

To develop a system analysis code, numerical methods, mesh management, equations of state, fluid properties, solid material properties, neutronics properties, pressure loss and heat transfer closure laws, and good user input/output interfaces are all indispensable. SAM leverages the MOOSE framework and its dependent libraries to provide JFNK solver schemes, mesh management, and I/O interfaces while focusing on new physics and component model development for advanced reactor systems. The developed physics and component models provide several major modeling features:

1. One-D pipe networks represent general fluid systems such as the reactor coolant loops.

2. Flexible integration of fluid and solid components, able to model complex and generic engineering system. A general liquid flow and solid structure interface model was developed for easier implementation of physics models in the components.

3. A pseudo three-dimensional capability by physically coupling the 1-D or 2-D components in a 3-D layout. For example, the 3-D full-core heat-transfer in an SFR reactor core can be modeled. The heat generated in the fuel rod of one fuel assembly can be transferred to the coolant in the core channel, the duct wall, the inter-assembly gap, and then the adjacent fuel assemblies.

4. Pool-type reactor specific features such as liquid volume level tracking, cover gas dynamics, heat transfer between 0-D pools, fluid heat conduction, etc. These are important features for accurate safety analyses of SFRs or other advanced reactor concepts.

5. An infrastructure for coupling with external codes has been developed and demonstrated.

An example of SAM simulation results of an SFR is shown in Figure 2. 

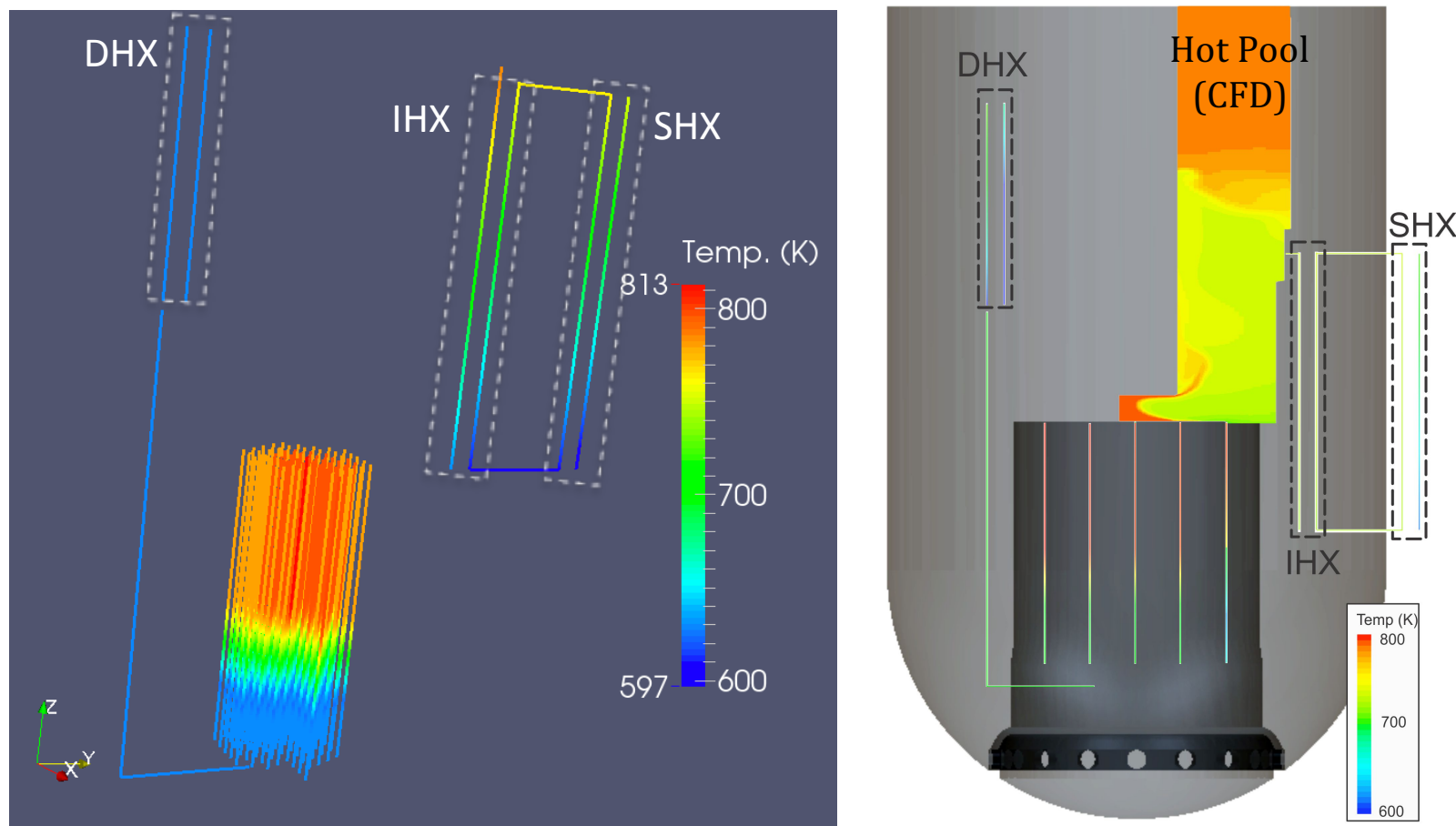

(a) SAM model with 61 core channels

(b) Coupled SAM and CFD code simulation Figure 2. SAM simulation results of an SFR. 


\section{One-D Modeling Approach}

As discussed in Chapter 1, current major system analysis codes only have 0-D models for thermal mixing and stratification in large enclosures. For example, SAS4A/SASSYS-1 code provides lumped-volume-based 0-D models that can give very approximate results and can only handle simple cases with one mixing source. The basic concept is based on that the lumped enclosure is made up of a small number of distinct temperature regions, separated by horizontal interfaces. Depending on the momentum and buoyancy of the jet flow into the large enclosures, well mixed case, two-zone with a negative buoyant jet case, two-zone with a positive buoyant jet case, and even more complex three-zone cases may form. The total jet entrainment, zone interface location, and average temperatures in each zone can be estimated by empirical correlations. Since the methods are based on scaled experimental data, using those models for SFR designs with different hot/cold pool configurations tends to result in large uncertainties.

The SAM 1-D modeling approach is based on similar concept that the large enclosure can be divided into arbitrary number of sub-volumes, separated by horizontal interfaces. The intervolume energy exchange can be modeled by both advection and flow mixing. To consider the flow mixing in 1-D flow, the energy conservation equation can be written as:

$$
\frac{\partial(\rho H)}{\partial t}+\frac{\partial\left(\left(\rho u+G_{m i x}\right) H\right)}{\partial z}=\nabla(k \nabla T)
$$

Where $G_{m i x}$ is the mixing mass flux, and $G_{m i x}=\rho u_{\text {mix }}$, in which $u_{\text {mix }}$ is the mixing flow velocity. Note that the mixing flow cannot be calculated using the 1-D modeling approach. Therefore, additional closure models for the mixing mass flux is needed, for which the highfidelity CFD simulations using LES and uRANS can assist in the closure model developments.

\subsection{Multiple 0-D Volume Modeling Approach}

A simplified version of the 1-D modeling approach is to model the large enclosure with multiple 0-D volumes, as shown in Figure 3. Note that it allows a 0-D volume connecting to another 0-D volume, which is implemented in SAM through StagnantVolume Component.

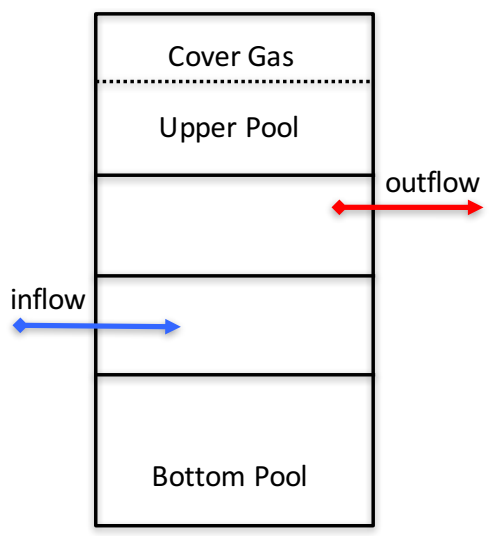

Figure 3. SAM Core Channel Model of EBR-II. 
StagnantVolume models a stagnant liquid volume, which has no connections to 1-D fluid components but is allowed to connect to a $0-\mathrm{D}$ volume or 1-D or 2-D heat structures for heat transfer. It is assumed that there is no net mass transfer between StagnantVolume and the connecting 0-D volumes. The governing equation of the energy conservation for the StagnantVolume can be given as:

$$
\frac{d(\rho V H)}{d t}+\sum_{i=1}^{n} \dot{Q}_{i}=0
$$

In which,

$\rho$ : average density of the StagnantVolume component;

$V$ : total volume of the component;

$H$ : average enthalpy of the volume component.

$t$ : time;

$n$ : the number of coupling heat transfer components;

$\dot{Q}$ : heat transfer with coupled heat structures or 0 -D volumes;

For convective heat transfer with heat structures,

$$
\dot{Q}=\int h_{\text {conv }}\left(T_{w}-T_{v o l}\right) d A
$$

In which,

$h_{\text {conv }}$ : convective heat transfer coefficient;

$T_{w}:$ structure wall temperature;

$T_{v o l}$ : volume temperature.

For heat transfer with other $0-\mathrm{D}$ volumes through thermal mixing,

$$
\dot{Q}=\dot{m}_{m i x} \Delta H
$$

In which,

$\dot{m}_{\text {mix }}$ : the effective mixing flow between 0 -D volumes;

$\Delta H$ : enthalpy differences between 0 -D volumes.

\subsection{Demonstration Simulation of EBR-II Transient}

The feasibility of the multiple 0 -D volume modeling approach was tested using the available an available EBR-II test BOP-302R.

\subsubsection{Model Description}

The EBR-II plant was a 62.5 MWth metallic fueled sodium fast reactor designed and operated between 1964 and 1994 by Argonne National Laboratory. During its operation, EBR-II was used for experiments designed to demonstrate the feasibility of passive safety in liquid metal reactors (LMR). The Shutdown Heat Removal Test program was carried out in EBR-II between 
1984 and 1986. The objectives of the program were to support the U.S. advanced LMR program, provide test data for validation of computer codes, and demonstrate passive reactor shutdown and decay heat removal in response to protected and unprotected transients. Supported by DOENE's Advanced Reactor Technology (ART) program, some of the EBR-II test data are recovered and organized into electronic databases.

The SHRT-17 and SHRT-45R, the most severe protected and unprotected loss of flow tests, haven been selected as benchmark problems under an International Atomic Energy Agency (IAEA) coordinated research project [26][27]. The benchmark specifications of the EBR-II tests are also being used to support code validation efforts during SAM development [28]. Major physics phenomena in the primary coolant loop during the protected-loss-of-flow transients were well captured in the SAM simulation. The multiple 0-D volume approach to model the effects of thermal mixing and stratification is demonstrated in SAM simulation of an unprotected loss of all heat rejection test, BOP-302R.

The BOP, or balance of plant, tests were a series of tests performed during the SHRT testing program to investigate transients where the primary sodium pumps did not trip. A variety of different BOP tests were performed ranging from tests where the control rod insertion depth fluctuated to other tests where the intermediate sodium electromagnetic pump was oscillated at various frequencies. BOP-302R was one of two loss-of-heat-sink tests where the intermediate sodium pump was tripped without scramming the control rods or tripping the primary pumps. This test was driven by increasing core inlet temperatures, which were a result of a diminished IHX heat rejection rate due to the lower intermediate sodium flow rates. BOP-302R was performed several hours after SHRT-45R and was initiated from full power and full flow. An EBR-II model, similar to the SAS4A/SASSYS-1 model described in Ref. [27], was developed for SHRT-45R and BOP-302R benchmark simulations, as shown in Figures 4 and 5. To simplify the input preparation, minor flow leakages are not modeled.

\section{Core Model}

The thermal-hydraulic performance of a reactor core is analyzed in SAM with a model consisting of a number of core channels. The channel model provides input to specify a single fuel pin and its associated coolant and structure. A single-pin channel represents the average pin in an assembly, and assemblies with similar reactor physics and thermal-hydraulic characteristics are grouped together.

Five single-pin channel types were created for the driver, partial driver, dummy, reflector, and blanket assemblies. The BOP-302R core models use 12 channels based on one of these five channel types to represent all 637 subassemblies. The safety, experimental, and control subassemblies are not modeled with their own channel types but rather are grouped into other channels. Table 1 and Figure 4 illustrate the channels in the EBR-II core model. Among them, six channels (with six different colors in Figure 4) represent: (1) the lumped fuel assembly groups of the driver fuel assemblies; (2) the high-flow driver fuel assemblies; (3) lumped channel for all dummy (K-type), partial driver (P-type), experimental, and control assemblies; (4) inner core reflector assemblies; (5) outer core reflector assemblies; and (6) blanket assemblies. Another six channels shown in red represent six individual assemblies with different types at locations 1A1, 2B1, 4C3, 6C4, 7A3, and 12E6. These subassemblies were modeled individually because they were among a subset of subassemblies whose outlet temperatures were measured. A 22-channel model is used in SAS4A/SASSYS-1, and the details can be found in Ref. [27]. 
Reactivity feedbacks are not modeled in these benchmark simulations as some important reactivity feedback mechanisms, such as the radial and axial core expansion, control rod drive line expansion, etc., cannot yet be modeled in SAM. Instead, the reactor power histories from the tests are directly applied in the simulation of the transients. Although these reactivity feedback mechanisms can be modeled in SAS4A/SASSYS-1, the same assumptions were used in both simulations for consistent comparisons of the thermal-hydraulics responses of the system throughout the transients.

Table 1. SAM lumped core channels

\begin{tabular}{|c|l|c|c|c|}
\hline $\begin{array}{c}\text { Channel } \\
\text { number }\end{array}$ & Representations & $\begin{array}{c}\text { Total Assembly } \\
\text { number }\end{array}$ & $\begin{array}{c}\text { Total Power } \\
(\mathrm{MW})\end{array}$ & $\begin{array}{c}\text { Total Flow } \\
(\mathrm{kg} / \mathrm{s})\end{array}$ \\
\hline 1 & Average Driver & 56 & 33.7 & 232 \\
\hline 2 & Average High Flow Driver & 18 & 10.3 & 67.5 \\
\hline 3 & $\begin{array}{l}\text { Lumped Channel, } \\
\text { K+X+P+Control }\end{array}$ & 28 & 8.66 & 77.3 \\
\hline 4 & Average Inner Reflector & 20 & 0.278 & 3.32 \\
\hline 5 & Average Outer Reflector & 180 & 0.855 & 11.2 \\
\hline 6 & Average Blanket & 329 & 4.50 & 64.5 \\
\hline 7 & 1A1, Half-Driver & 1 & 0.332 & 3.81 \\
\hline 8 & 2B1, K016 & 1 & 0.0177 & 0.685 \\
\hline 9 & 4C3, Driver & 1 & 0.692 & 4.86 \\
\hline 10 & 6C4, Driver & 1 & 0.598 & 3.71 \\
\hline 11 & 7A3, Reflector & 1 & 0.0149 & 0.165 \\
\hline 12 & 12E6, Blanket & 1 & 0.0268 & 0.163 \\
\hline Total & & 637 & 60.0 & 469 \\
\hline
\end{tabular}




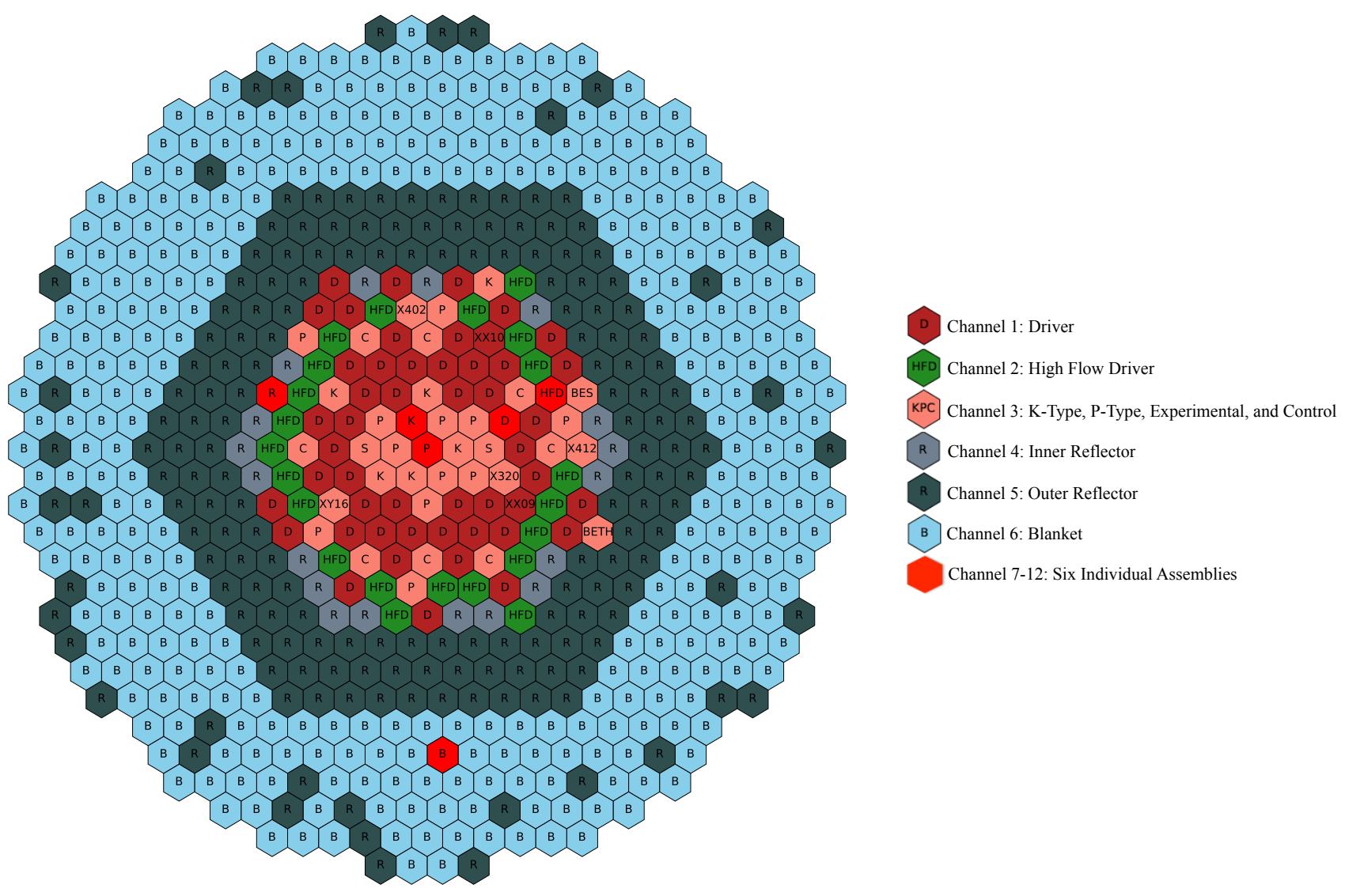

Figure 4. SAM Core Channel Model of EBR-II.

\section{Coolant System Model}

Figure 5 illustrates the EBR-II primary system model used in the SAS4A/SASSYS-1 simulations. A similar model is used in the SAM simulations except that the minor flow leakage paths were not modeled. The two primary pumps draw sodium from the cold pool and feed the high- and low-pressure flow paths. Sodium flows through the high-pressure inlet piping (e.g. E14 $\rightarrow \mathrm{E} 15 \rightarrow \mathrm{E} 16)$ and is discharged into the high-pressure inlet plenum before flowing up through the inner core channels. Sodium flowing through the low-pressure inlet piping (e.g. $\mathrm{E} 17 \rightarrow \mathrm{E} 18 \rightarrow \mathrm{E} 19 \rightarrow \mathrm{E} 20 \rightarrow \mathrm{E} 21)$ is discharged into the low-pressure inlet plenum before flowing up through the outer core channels. The inner core channels represent the first seven rows of subassemblies and the outer core channels represent the remaining subassemblies in rows 8-16. At steady state, the mass flow rates through the inner core and outer core subassemblies are approximately $390 \mathrm{~kg} / \mathrm{s}$ and $70 \mathrm{~kg} / \mathrm{s}$, respectively.

The inner and outer core channels both discharge into the outlet plenum, which mixes the discharged sodium before it enters the Z-Pipe. The Z-Pipe is a double-walled pipe that contains the auxiliary EM pump. Sodium leaving the Z-Pipe flows through the intermediate heat exchanger before discharging into the cold pool. 


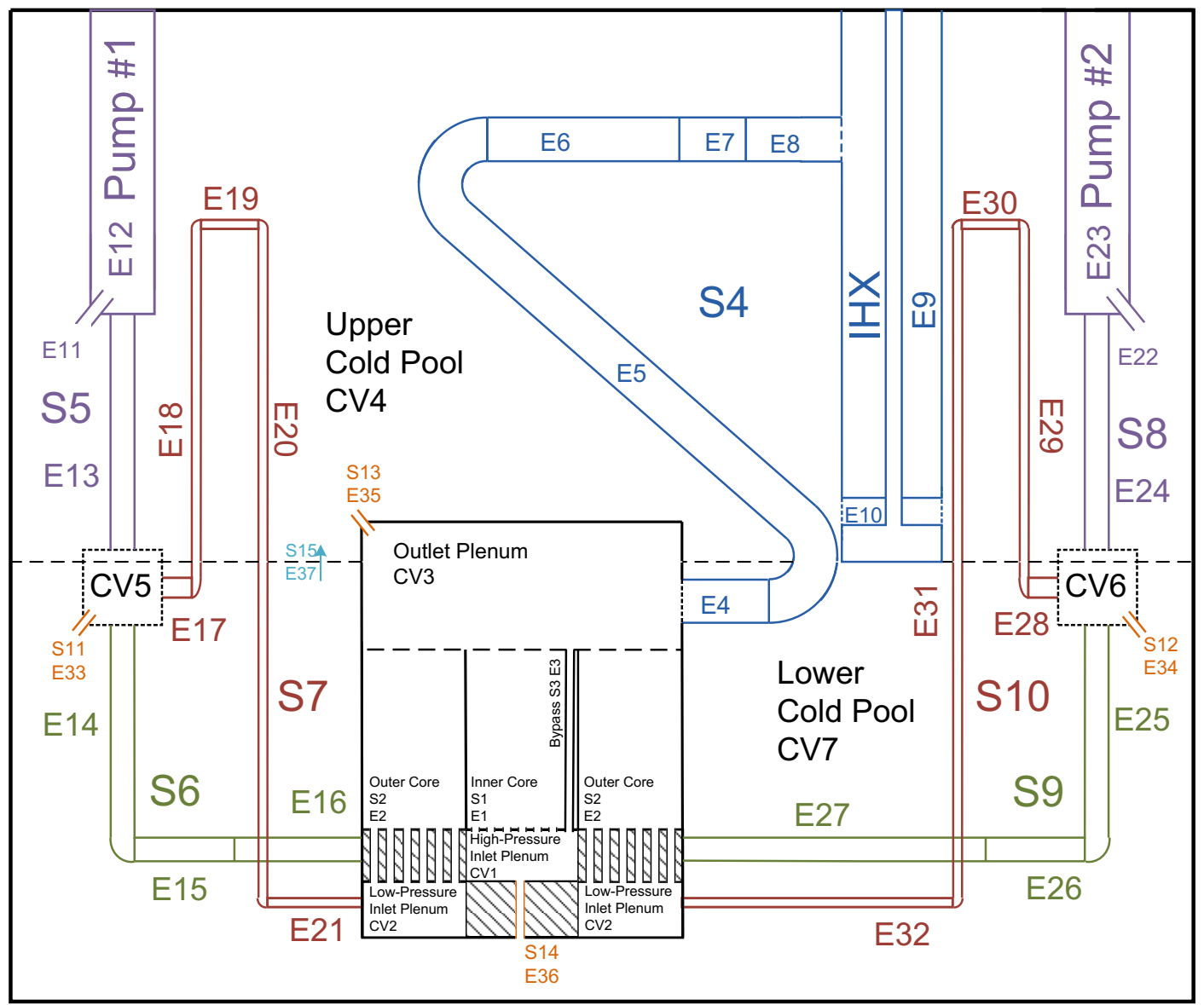

Figure 5. EBR-II Primary Sodium System Model.

\subsubsection{Simulation Results}

BOP-302R was a loss of heat sink test where the intermediate sodium pump was tripped without scramming the control rods or tripping the primary pumps. This test was driven by increasing core inlet temperatures, which were a result of the diminished IHX heat rejection due to the lower intermediate sodium flow rates. Strong thermal stratification is expected in the primary vessel (cold pool), where the IHX outlet and the pump inlets are located in the upper part of the vessel.

To correctly predict the core inlet temperature, the thermal stratification in the cold pool needs to be properly considered. This is accounted for in the SAM simulation with a two-volume pool model, in which the upper volume connects with the main primary pumps and the IHX, and the lower volume is stagnant but the mixing flow with the upper volume and the convective heat transfer with the immersed piping walls are considered. Similar modeling approaches were also adopted in the SAS4A/SASSYS-1 model. In the SAM BOP-302R simulation, the mixing flow between the upper and lower cold pool is assumed to be $50 \%$ of the primary core flow rate.

Simulation results of the BOP-302R test are shown in Figures 6 - 12. Very good agreement was found among the SAM and SAS simulations and the test results. The reactor core power and the instantaneous heat removal rate of IHX are shown in Figure 6. As discussed above, this 
transient is initiated by a complete loss of forced coolant flow in the intermediate loop, which resulted in a diminished IHX heat rejection rate. The reactor power is slowly reduced due to various reactivity feedback mechanisms. Throughout the transient, the reactor power is always higher than the IHX heat removal rate. For a short period, the IHX secondary side temperature is higher than the primary side, which results in heat transfer from the intermediate loop to the primary loop.

SAM predictions of the plena temperatures during BOP-302R test are shown in Figure 7. The upper cold pool temperature increases rapidly at the beginning of the transient due to the loss-ofcooling in the IHX. As the primary loop coolant flow rate is largely unchanged throughout the transient, the core outlet plenum temperature drops with the decrease of the core power. As the IHX primary outlet temperature decreases and the continuous mixing between the upper and lower cold pool, the upper cold pool temperature decreases eventually after reaching a peak. The temperature responses in the high- and low-pressure core inlet plena are similar to the upper cold pool, and the temperature at the low-pressure inlet plenum is slightly lower due to the heat loss to the lower cold pool through the long piping. The lower cold pool temperature response is much slower compared to other volumes, as it does not directly participate in the primary coolant flow loop. Eventually all plena temperatures became very close to each other as the lower cold pool was heated up.

The comparisons of high- and low-pressure inlet plena temperatures from the SAM and SAS simulations and the test results are shown in Figure 8 and Figure 9. Very good agreement was achieved for both the initial heat up rates and the later pseudo-equilibrium states. It is demonstrated that the thermal stratification in the cold pool during the test can be modeled with a relatively simple multiple 0 -D volume model. Note that the mixing flow rates between 0 -D volumes are crucial to accurately model the heat transfer between 0 -D volumes, and the mixing flow rates can be derived from uRANS-based CFD simulations.

The Z-Pipe inlet and IHX primary inlet temperature responses are shown in Figure 10 and Figure 11. As the primary loop coolant flow rate is largely unchanged at full flow, the coolant temperature rise across the reactor core is very small. The core outlet temperature continues dropping as the reactor power decreases. The Z-Pipe inlet and IHX primary inlet temperature responses follow the same response. This is expected because the heat loss through the Z-Pipe walls is negligible during high flow conditions. Very good agreement was found among the two code simulations and the test results.

The core outlet temperature of the driver fuel subassembly 6C4 is shown in Figure 12. The transient trends among the experiment and the two simulations are very similar, but the initial increase of core outlet temperature was not observed in the test results. It is suspected again that the mixing between the subassembly $6 \mathrm{C} 4$ and the adjacent low power subassemblies reduced its outlet temperature measurement in the test. Very similar results are found between the SAM and SAS simulations. 


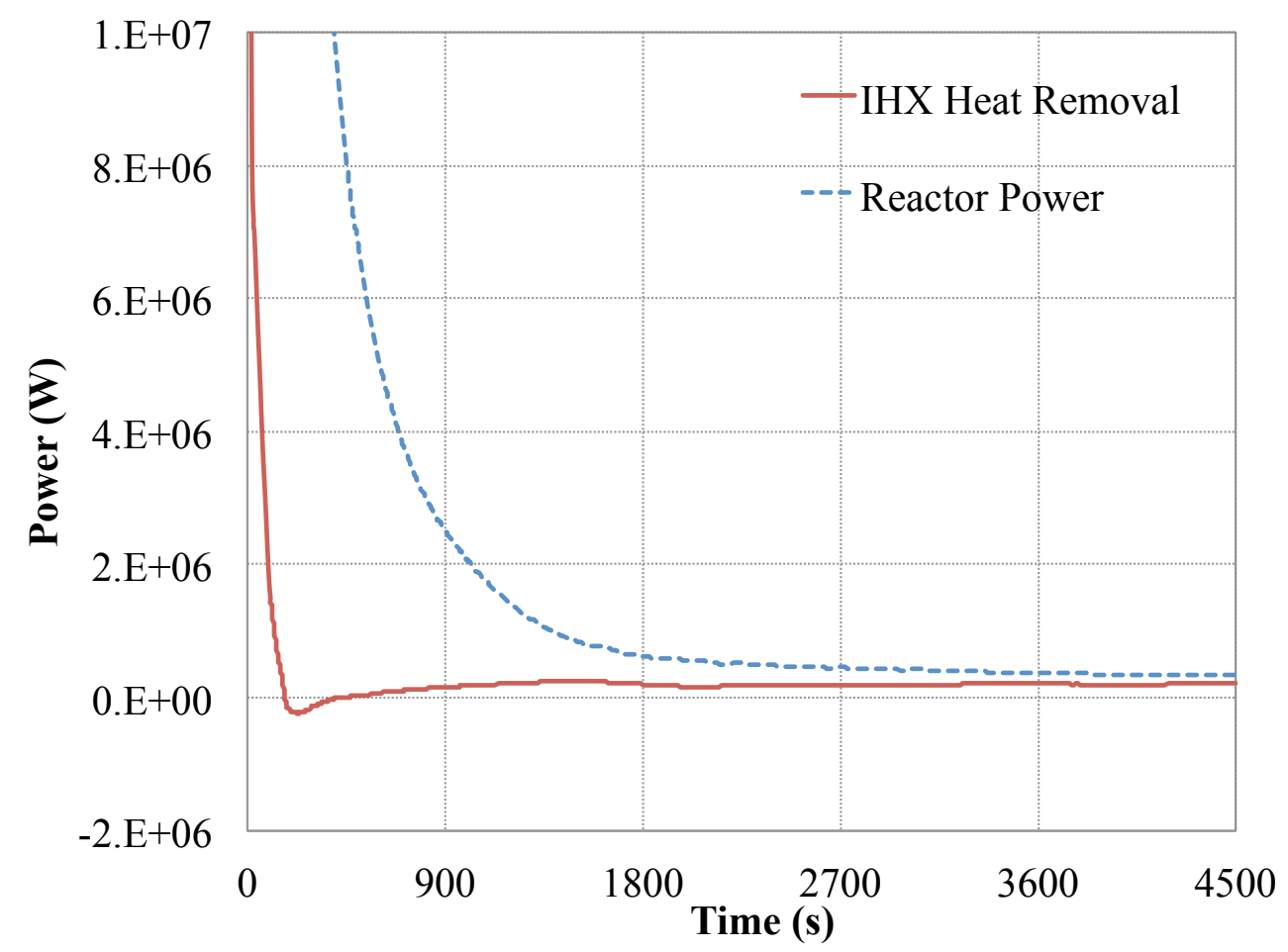

Figure 6. Reactor power and IHX heat removal rate during BOP-302R test

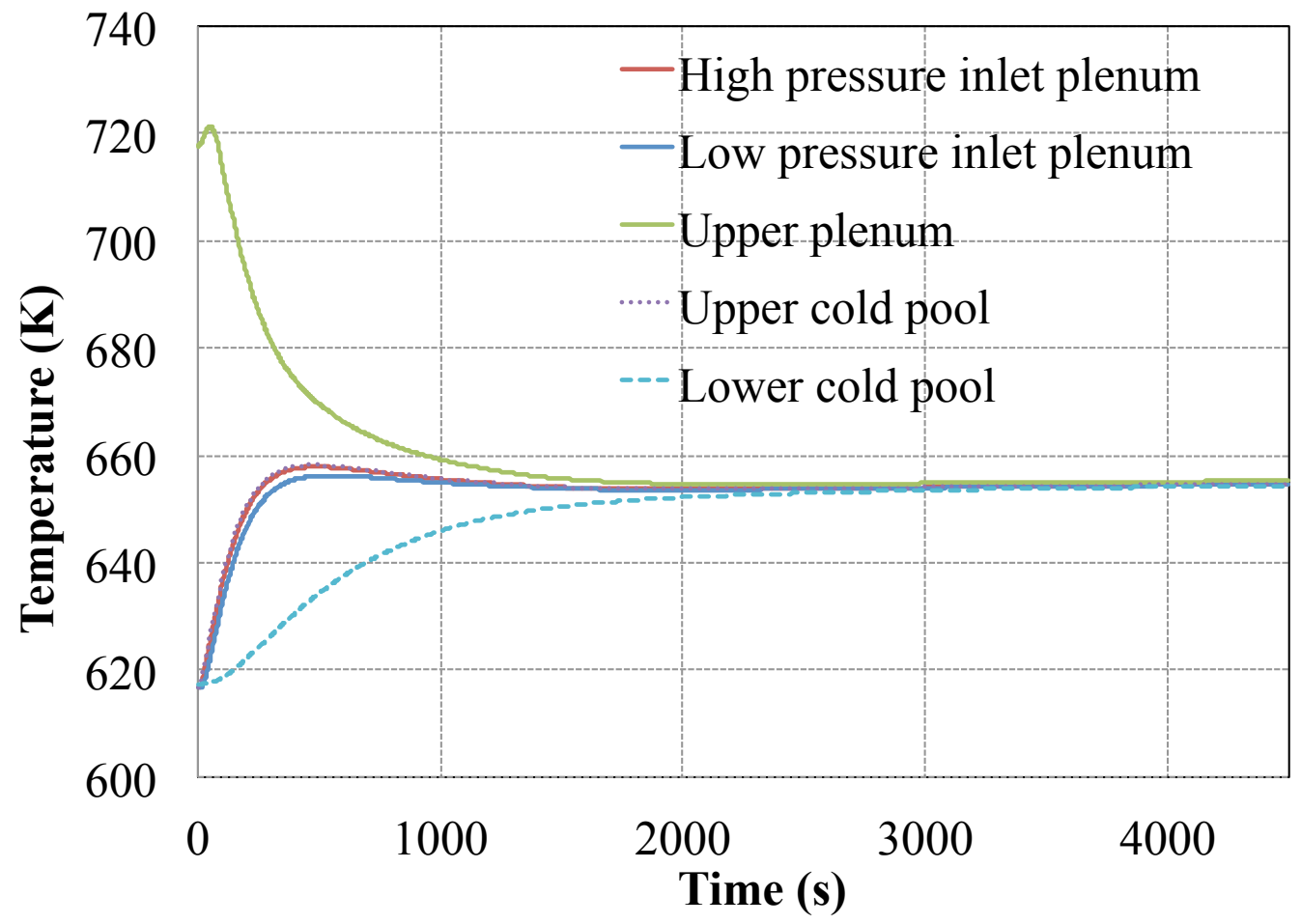

Figure 7. SAM predictions of plena temperatures during BOP-302R test 


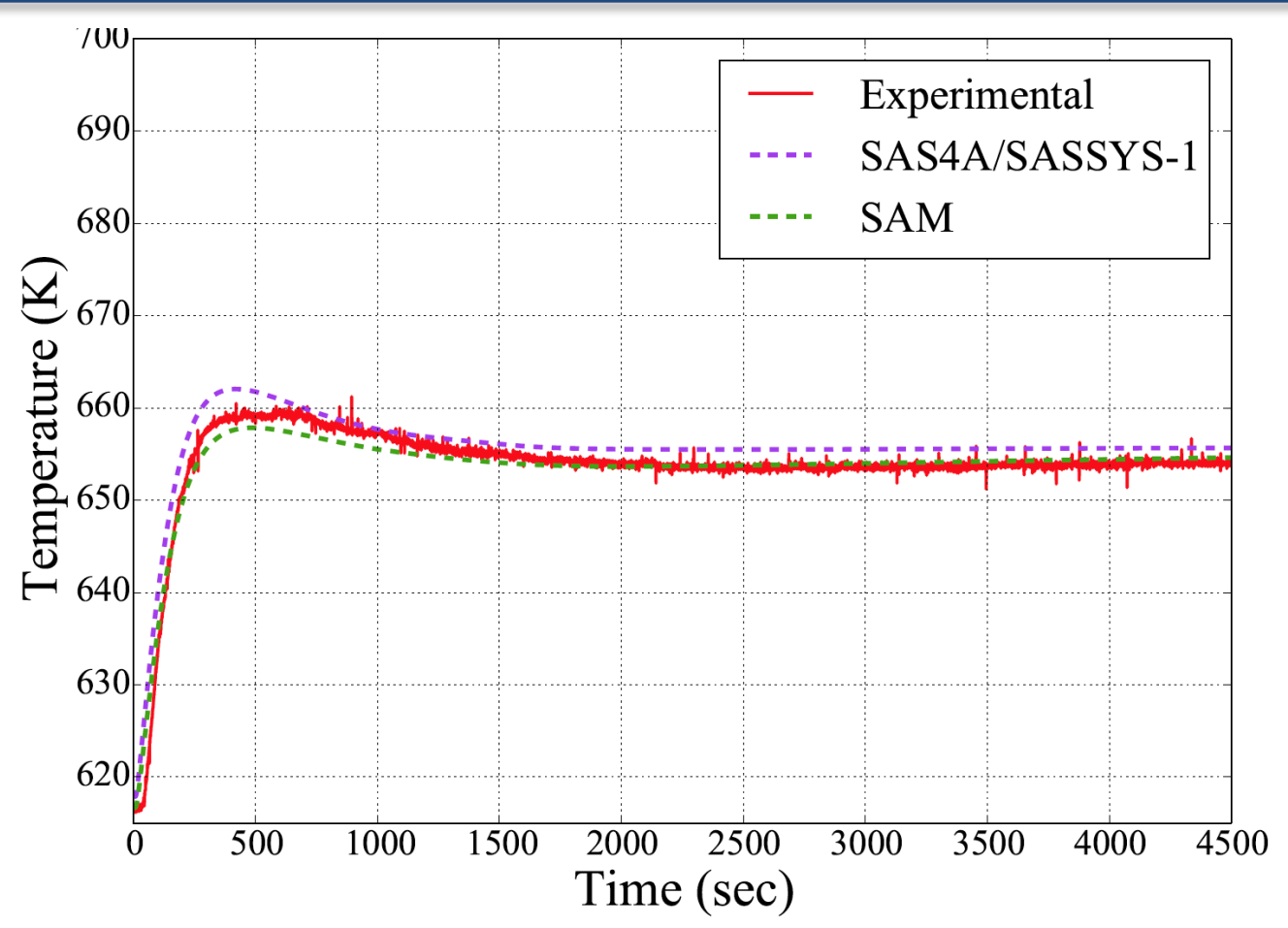

Figure 8. High-pressure inlet plenum temperature during BOP-302R test

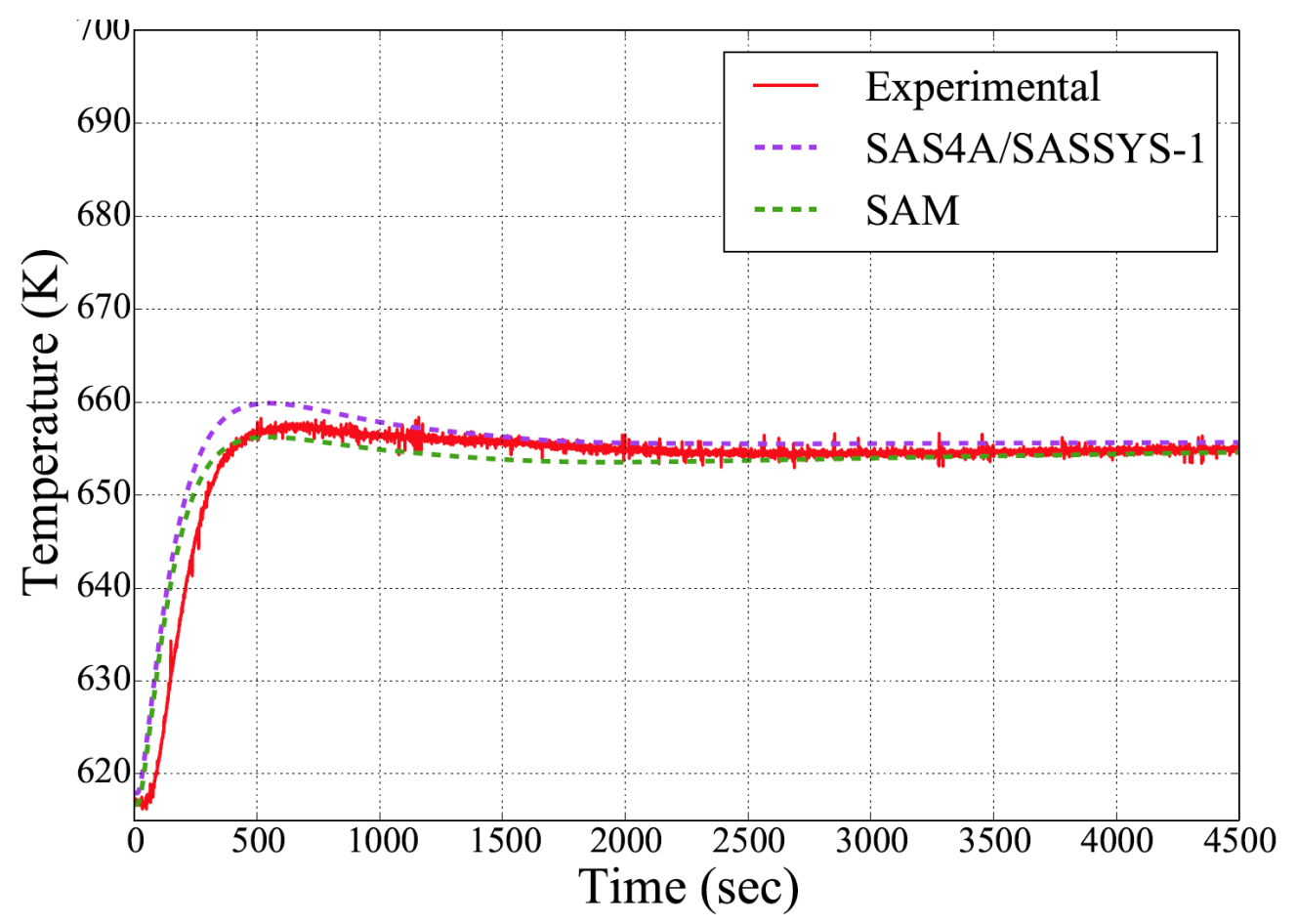

Figure 9. Low-pressure inlet plenum temperature during BOP-302R test 


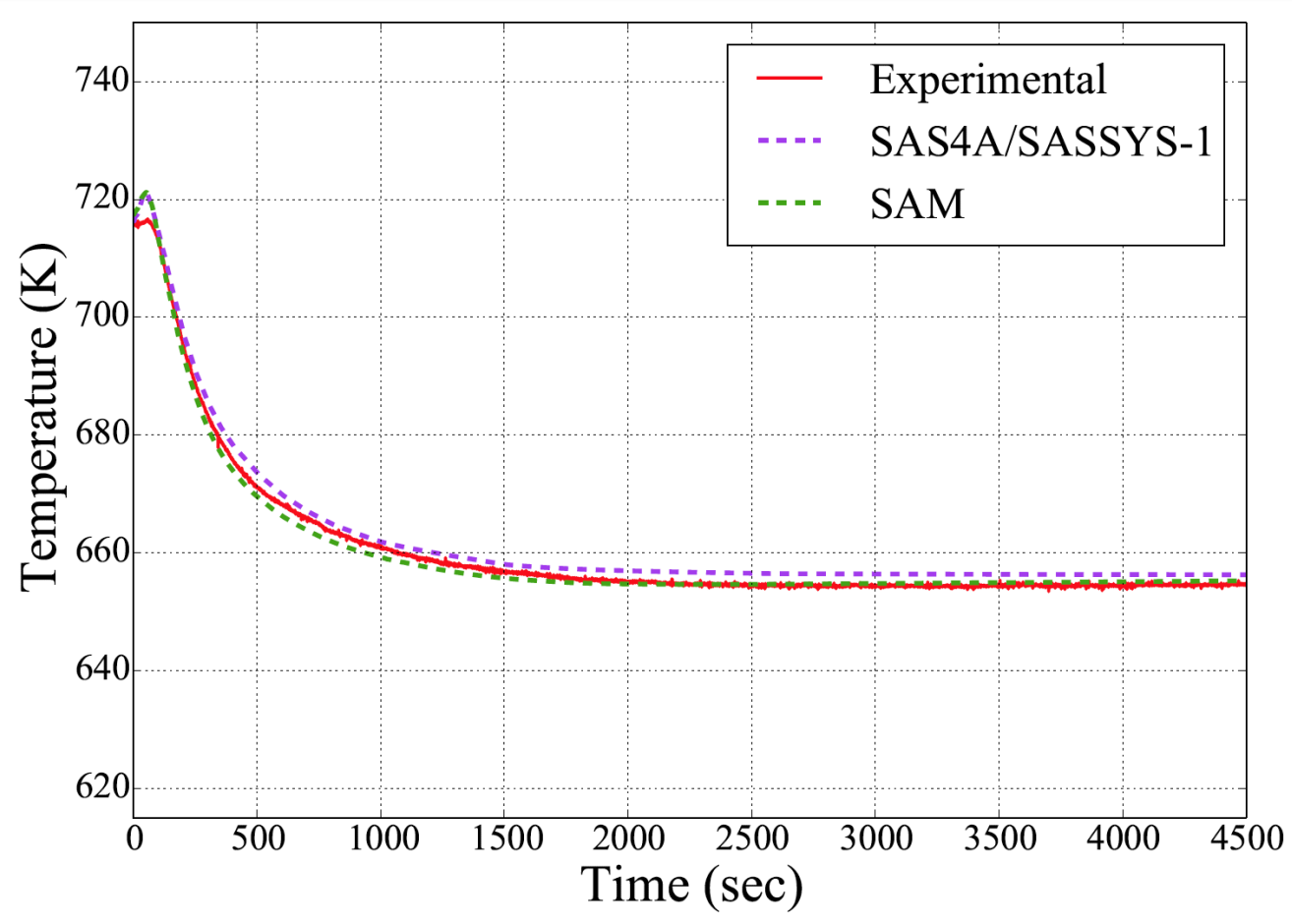

Figure 10. Z-Pipe inlet temperature during BOP-302R test

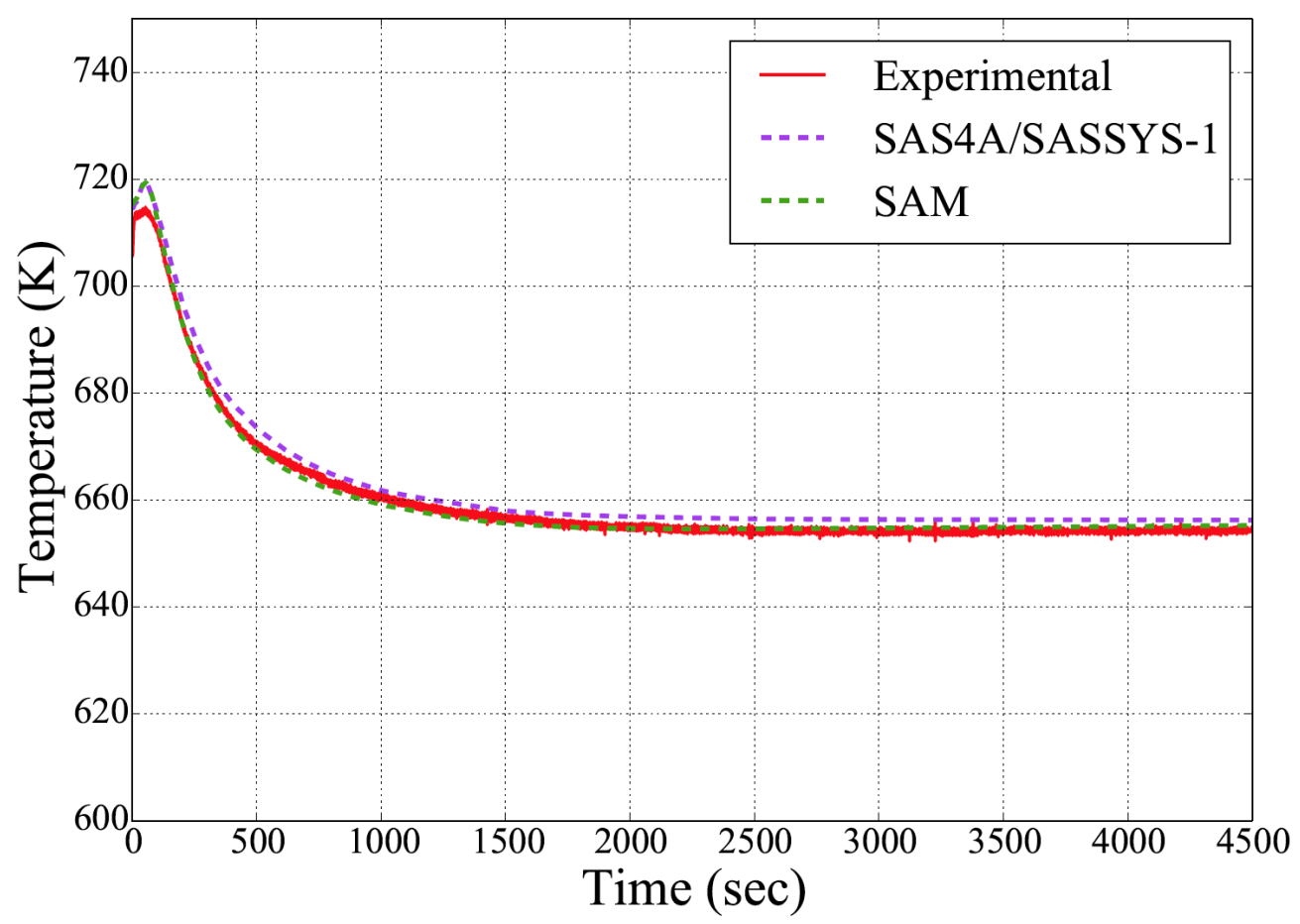

Figure 11. IHX primary inlet temperature during BOP-302R test 


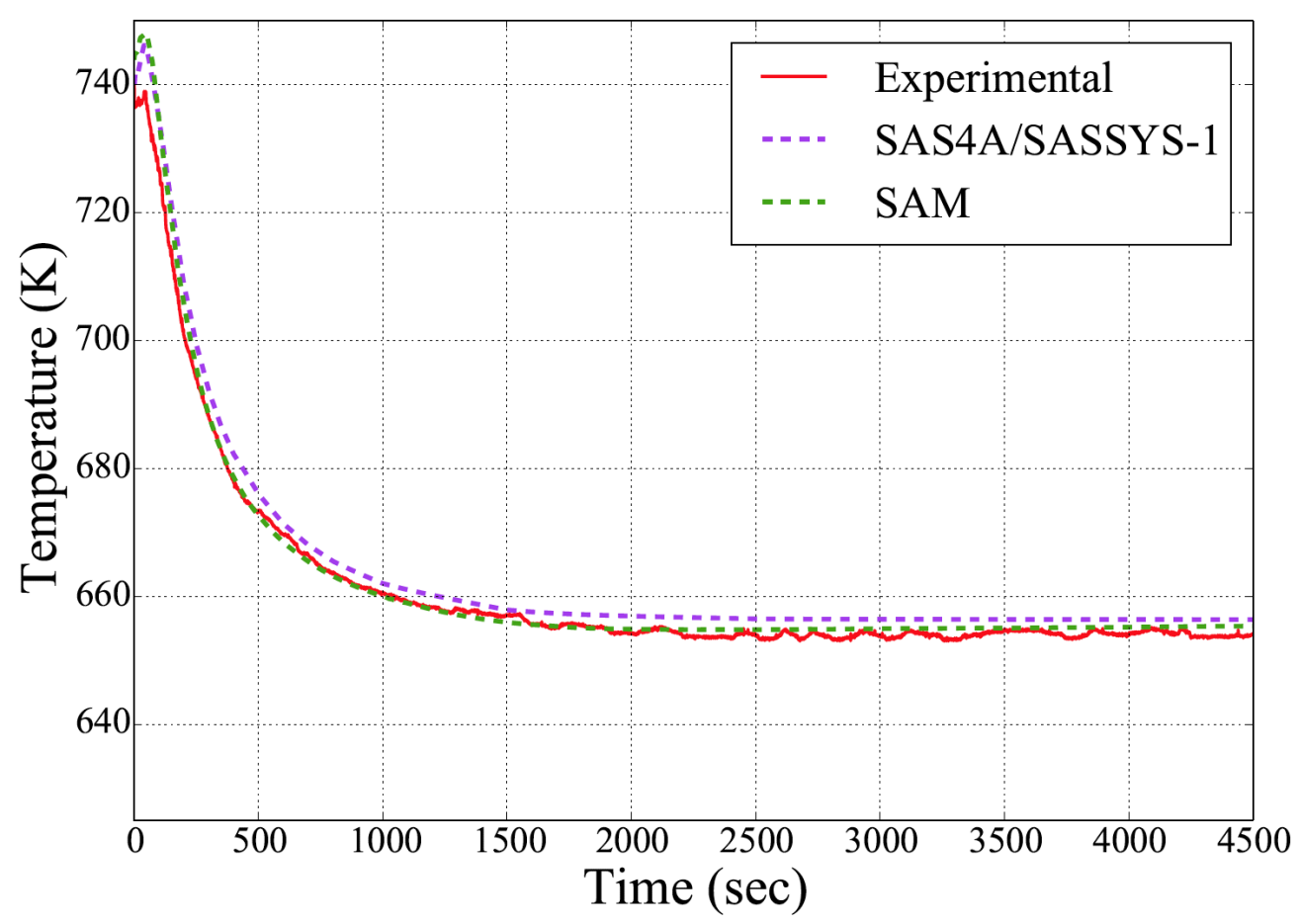

Figure 12. Subassembly 6C4 outlet temperature during BOP-302R test

\subsection{CFD Simulation of EBR-II BOP302R}

The benchmark simulation of the above EBR-II unprotected loss of heat rejection test (BOP$302 \mathrm{R}$ ) have been successfully performed. Focused on the thermal-hydraulics responses of the system throughout the transients in which the reactor power history was specified in the input model, very good agreement was found among the code simulations and the test results. The high- and low-pressure inlet plena temperatures from the SAM simulation agreed very well with the test for both the initial heat up rates and the later pseudo-equilibrium states, which demonstrated that the thermal stratification in the cold pool was correctly accounted for by the simple two-volume cold pool model. However, the assumption of the mixing flow (50\% of the primary core flow rate) between the upper and lower cold pool has strong influence to the transient simulation. Therefore, its validity needs to be examined using CFD simulations.

The CFD model of the EBR-II cold pool has been developed from a previous study [29] of a coupled system/CFD code solution of EBR-II transient. 3D geometry of important components such as IHX, primary pump, Z-Pipe, high and low pressure piping, were built in STAR-CCM+ 3D CAD module. Details of the CFD model including the mesh can be found in Ref. [29].

The CFD model of the EBR-II cold pool was used to simulate the transient response in BOP$302 \mathrm{R}$, an unprotected loss of heat rejection test. IHX outlet temperature from the stand-alone SAM simulation was provided as boundary conditions in the CFD model, as shown in Figure 14. The IHX outlet velocity was kept constant as the primary coolant pump kept running throughout the whole transient. 
Steady CFD simulations were performed first before initiating the loos-of-heat transient for the BOP-302R transient. The vertical velocity distributions in the cold pool before the transient is shown in Figure 15. Strong jet impinging effects were found when the IHX outlet flow entered into the cold pool, indicating strong mixing effects between the IHX outlet jet and the cold pool during the steady state operating conditions. After the horizontal jet hit the reactor vessel wall and the core shielding wall, part of the fluid then flowed upward, while the other portion flowed downward. At a horizontal cross plane near IHX bottom, downward flow was observed near the reactor vessel wall and the core shielding wall near IHX, while upward flow was observed near the IHX shell and the reactor vessel wall far away from IHX.

The evolutions of vertical velocity and temperature distributions in the cold pool during BOP-302R transient are shown in Figure 16 and Figure 17. The IHX outlet flow velocity remains very high throughout the transient, therefore the initial jet impinging phenomena maintained at the reactor vessel wall and the core shielding outer wall near IHX. Immediately after the transient, the IHX outlet temperature was increased significantly higher than the pool temperature, creating a positive buoyant force for the jet. At $t=50 \mathrm{~s}$ and $t=100 \mathrm{~s}$ downward flow at the jet imping walls disappeared. As hot fluid continued flowing into the hot pool, thermal stratification was formed in the upper part of the cold pool. When the inlet flow temperature started dropping while the upper part of the hot pool temperature started increasing, the positive buoyancy force to the jet would reduce. At $t=300 \mathrm{~s}$, the jet impinging effects were prominent again as the temperature differences between the jet and upper part of the cold pool became small. As the transient continued, the thermal stratification interface continued propagating downward. At $t=2000 \mathrm{~s}$, the vertical velocity distributions became similar to the steady state distributions. 


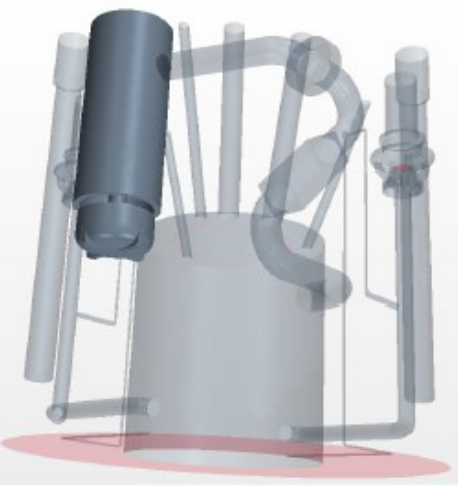

(a) CAD geometry IHX

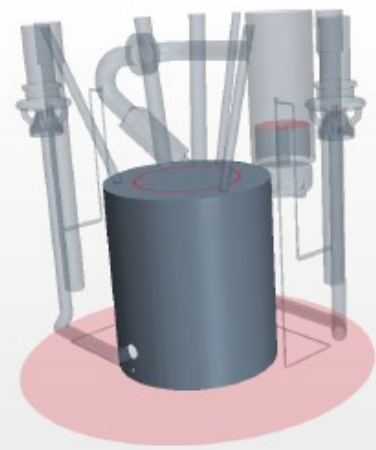

(c) CAD geometry shielding

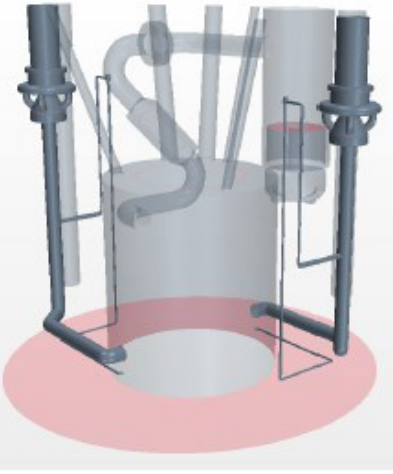

(b) CAD geometry primary pumps

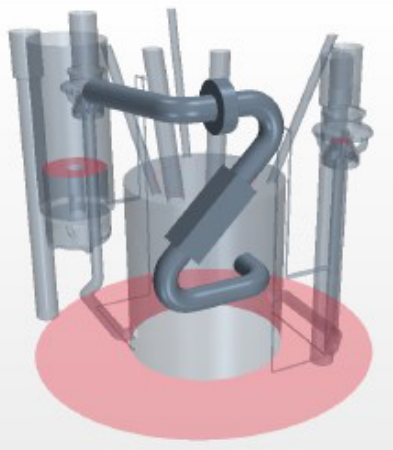

(d) CAD geometry Z-Pipe

Figure 13. EBR-II geometries with STAR-CCM+ CAD modeller

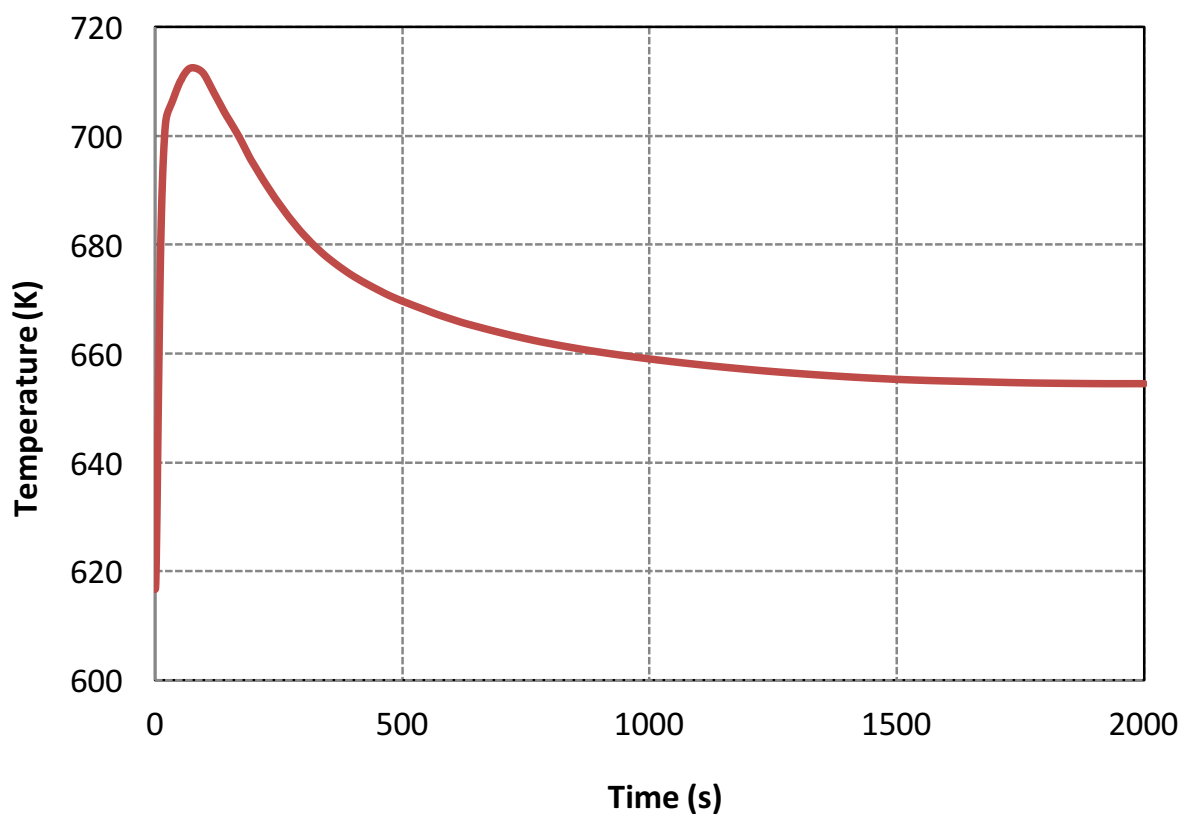

Figure 14. IHX outlet temperatures during the BOP-302R test 


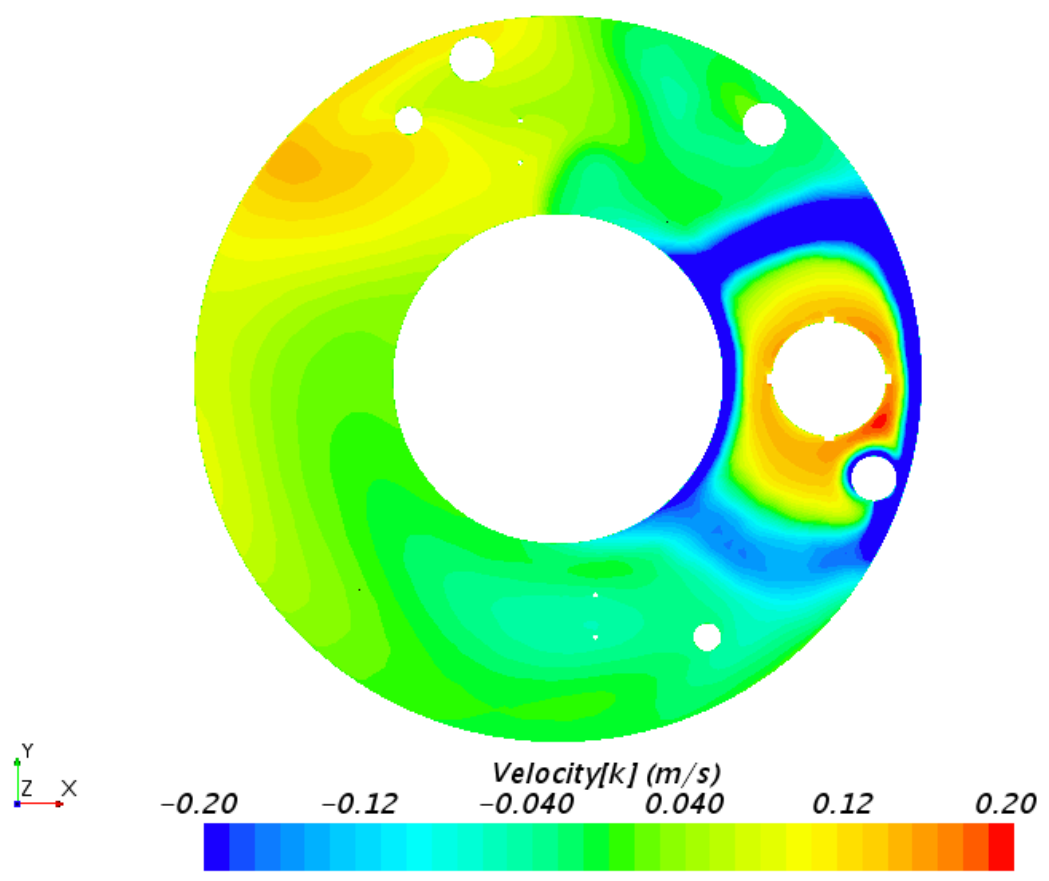

(a) Horizontal cross plane near IHX bottom

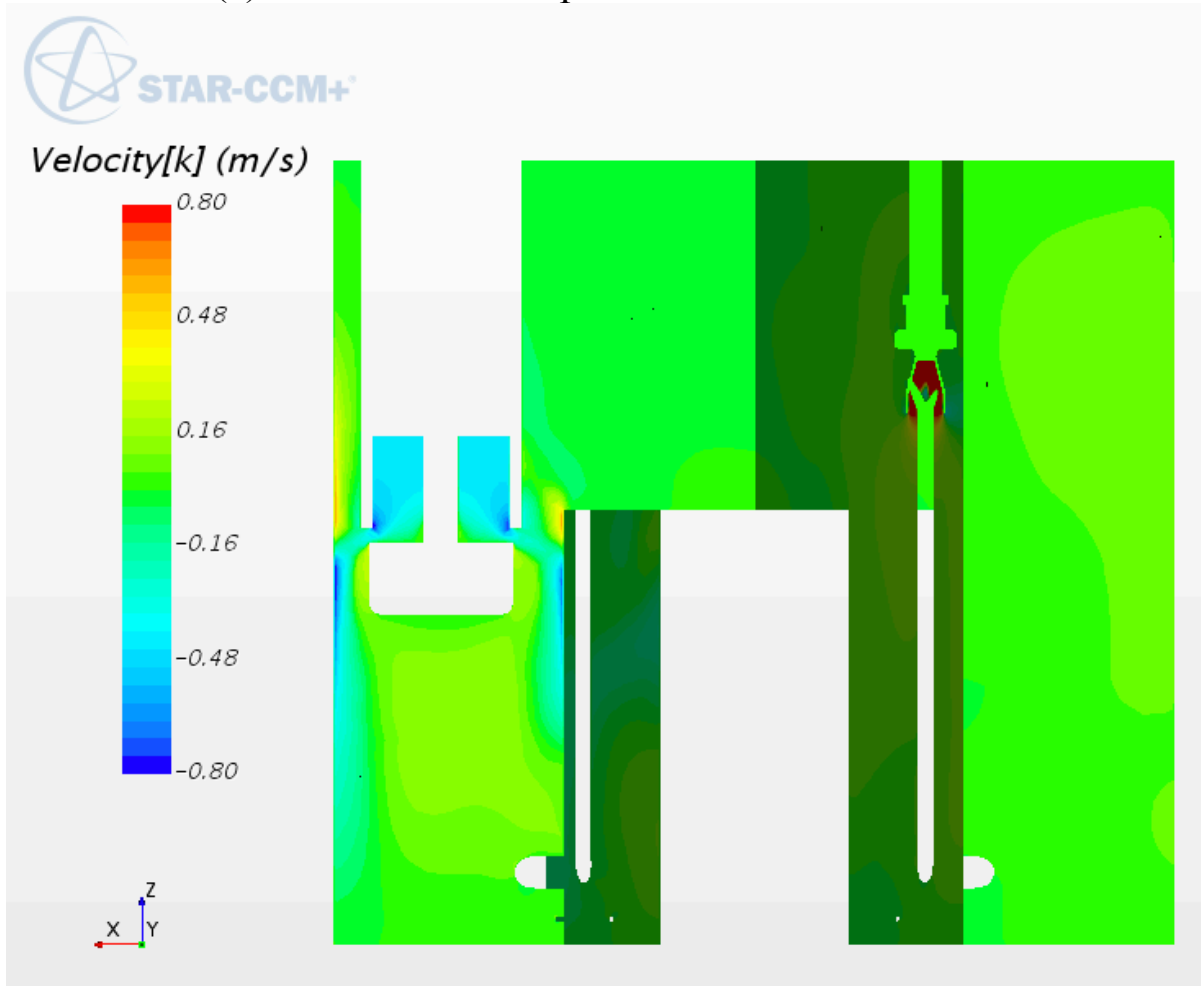

(b) Vertical cross planes (IHX and Pump view)

Figure 15. CFD prediction of the steady-state velocity distributions before BOP-302R transient 


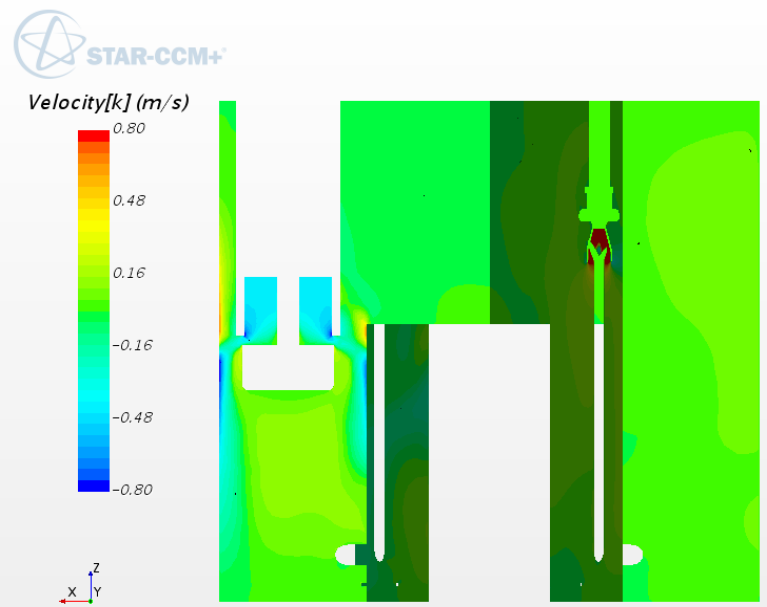

Solution Time $O(s)$

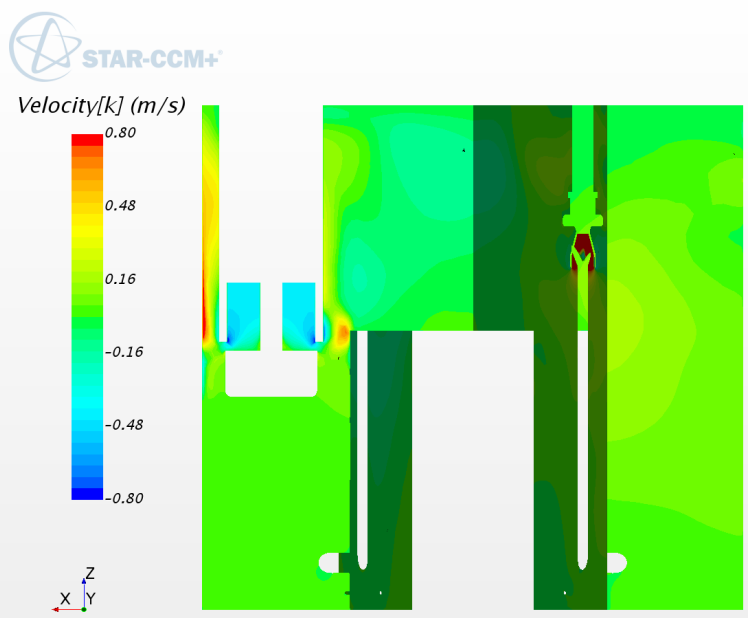

Solution Time 100 (s)

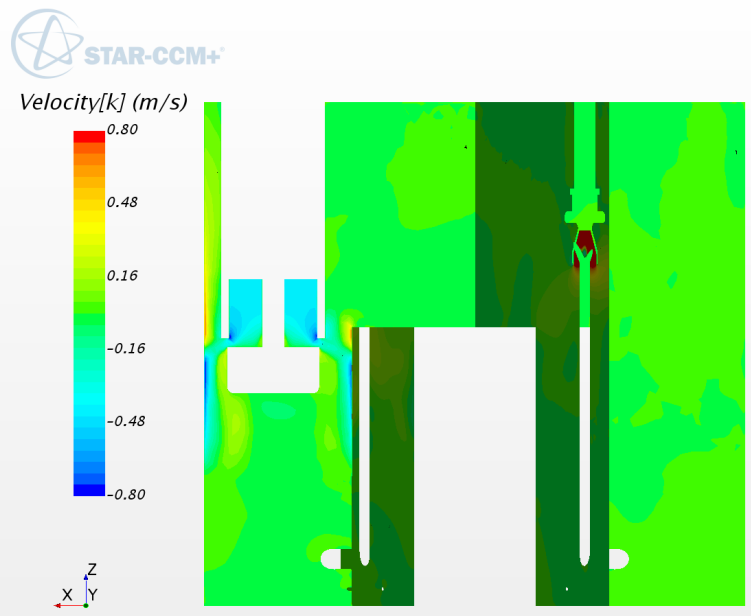

Solution Time 500 (s)

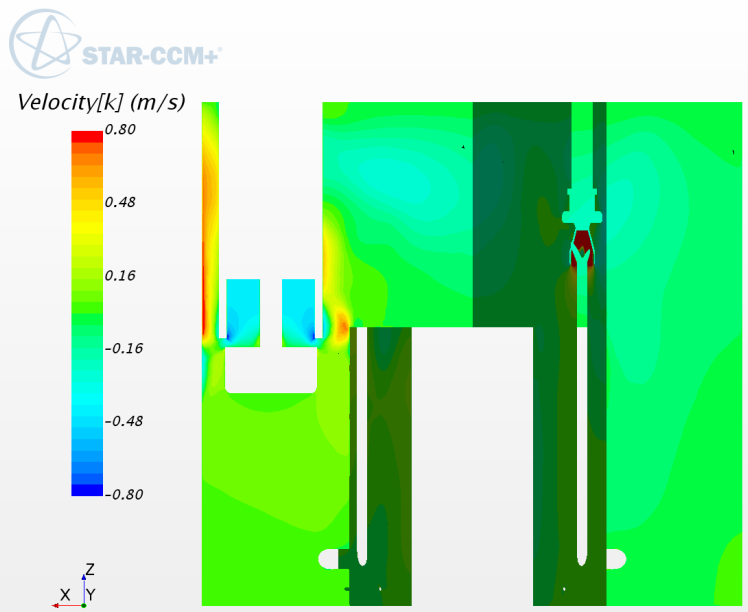

Solution Time 50 (s)

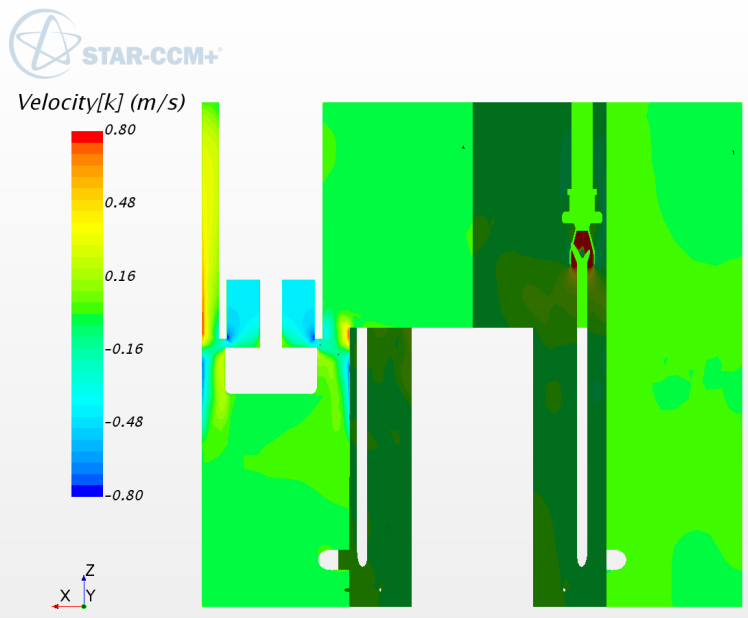

Solution Time 300 (s)

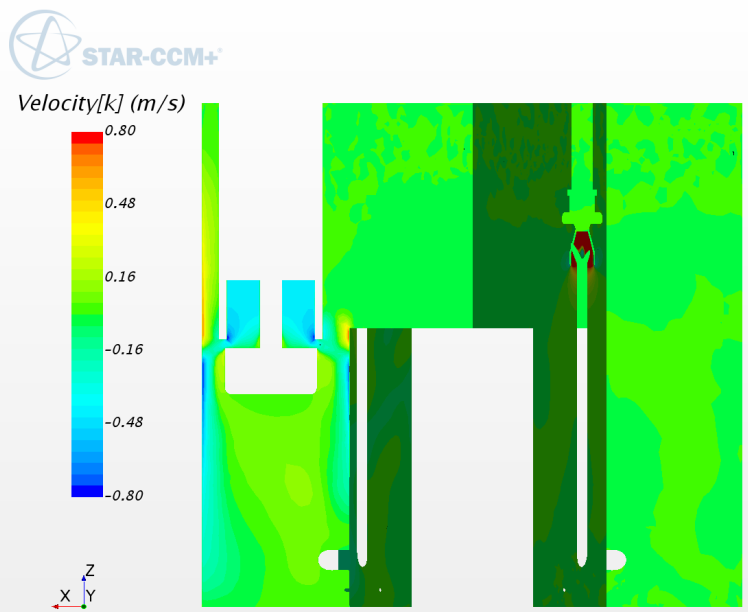

Solution Time 2000 (s)

Figure 16. CFD prediction of the velocity distributions during BOP-302R transient 


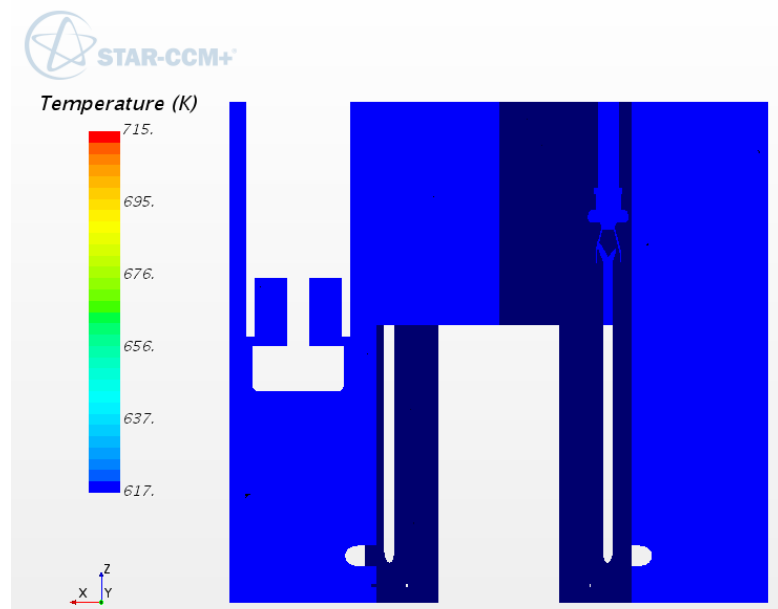

Solution Time $O$ (s)

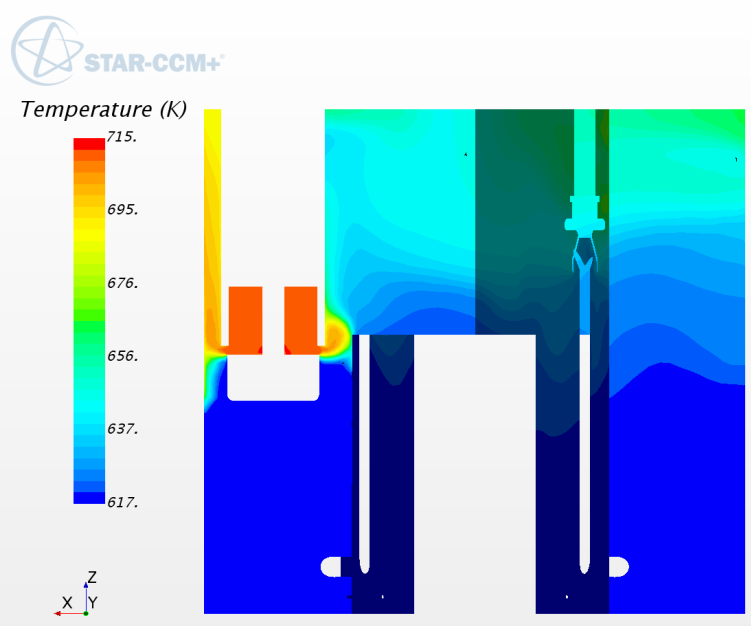

Solution Time 100 (s)

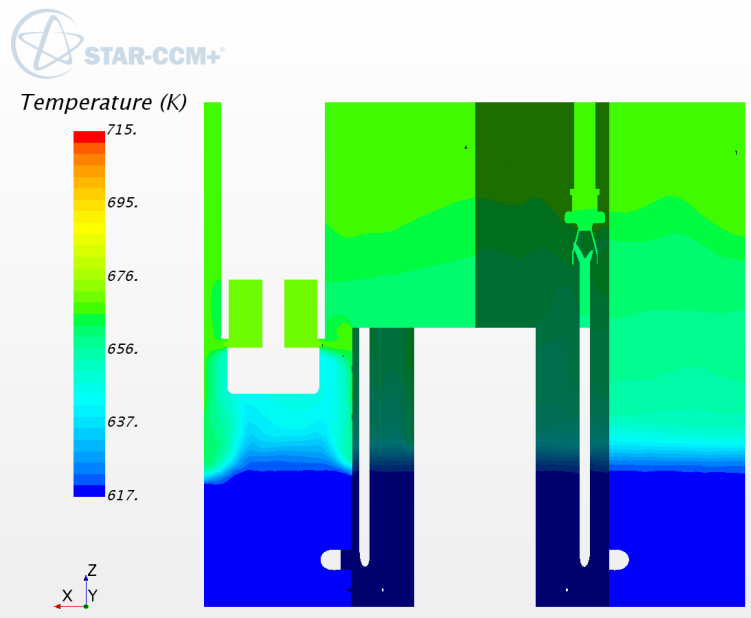

Solution Time 500 (s)

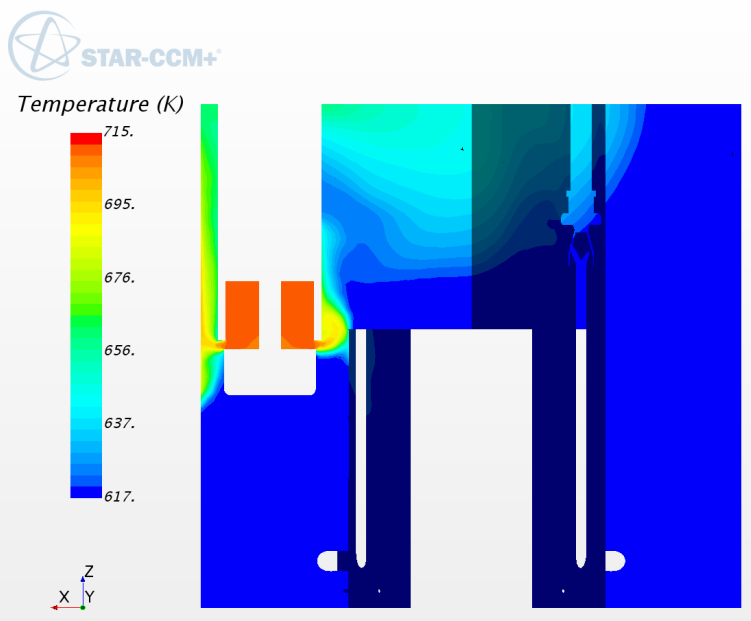

Solution Time 50 (s)

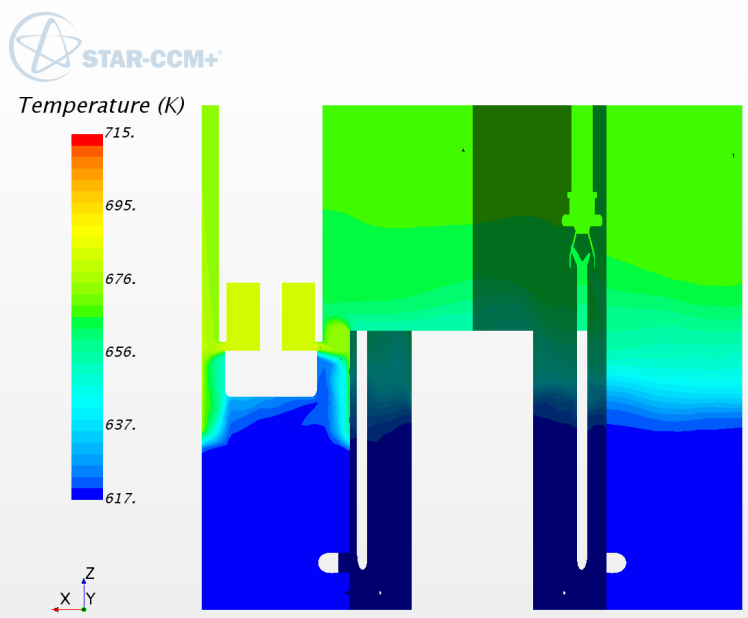

Solution Time 300 (s)

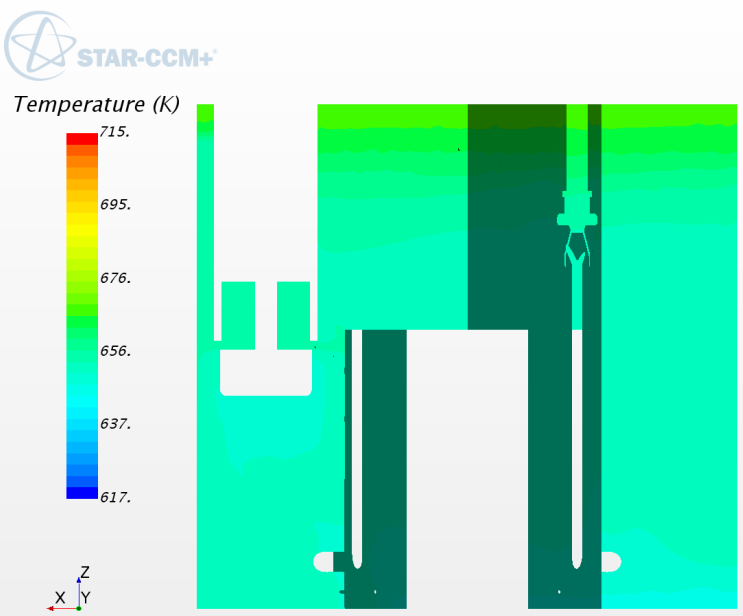

Solution Time 2000 (s)

Figure 17. CFD prediction of the temperature distributions during BOP-302R transient 
As discussed above, the thermal stratification in the cold pool strongly influenced the transient response in BOP-302R test. In the SAM stand-alone simulation of the test, a twovolume pool model was applied, in which the mixing flow (50\% of the normal core flow rate) between the upper and lower cold pool is tuned to best match the test results. To check the validity of this assumption, the u-RANS based CFD simulation results were used to derive the mixing flow rates throughout the whole transient.

For the horizontal cross planes (interfaces) below the IHX outlet, the net mass flow across each plane is almost zero. However, large circulations were observed during the transient, and the mixing flow across these interfaces can be derived from CFD simulation results:

$$
\begin{aligned}
& \dot{m}_{\text {total }}=\sum_{i}\left(\rho A v_{k}\right)_{i}=0 \\
& \dot{m}_{\text {mix }}=\frac{1}{2} \sum_{i}\left(\rho A\left|v_{k}\right|\right)_{i}
\end{aligned}
$$

In which, $v_{k}$ is the velocity in the vertical direction; $i$ is the index of all cells of the interface.

The mixing flow rates at various horizontal cross planes during the transient are shown in Figure 18. High mixing flow rates at these cross planes were observed before transient started, caused by large flow circulation resulted from the IHX outlet jet impinging to the reactor vessel wall and the core shielding outer wall. Immediately after the transient, the IHX outlet temperature was increased significantly higher than the pool temperature, creating a positive buoyant force for the jet. The buoyant effects to the jet and the thermal gradients in the cold pool resulted in increased mixing flow rates in the upper part of the cold pool, and decreased mixing flowrates in the lower part of the cold pool. After 200s into the transient, the IHX outlet temperature started decreasing again, and the upper part of the cold pool was heated up. The jet impinging effects were prominent again as the temperature differences between the jet and upper part of the cold pool became small. After 1000s, the flow circulation pattern became similar to the steady state conditions, and the mixing flow rates at different interfaces remained almost constant.

Mixing flow rate in a pool is often dependent on Richardson number, a dimensionless number that expresses the ratio of the buoyancy force to the flow gradient term. For buoyancy jet,

$$
R i=\frac{\left(\rho_{a}-\rho_{0}\right) g d}{\rho_{a} U_{0}^{2}}
$$

In which, $\rho_{o}$ is the jet temperature; $\rho_{a}$ is the ambient temperature. Because the major downward flow is caused by horizontal jet impinging, the characteristic length can be defined as the depth between the interface and the IHX outlet, $d=0.606 \mathrm{~m}$.

The mixing flow rate and the jet Richardson number at the horizontal cross plane at IHX shell bottom during the transient are shown in Figure 19. The relationship between mixing flow at interfaces and $R i$ is not obvious, and may be difficult to extrapolate. A possible reason is that the mixing flow at the interfaces is dependent on the evolution of the circulation patterns and the temperature distributions in the pool, which is time-dependent. 
Although the mixing flow rate is hard to be correlated with the jet Richardson number and other macroscopic parameters, it is clearly shown that it was not constant throughout the transient, and the magnitude is much higher than the $50 \%$ of normal core flow rate $(\sim 235 \mathrm{~kg} / \mathrm{s})$. This indicate that the assumption on the mixing flow rate used in stand-alone SAM simulation of BOP-302 test is not valid, even though the simulation results matched well with the experimental results. Further study is needed to develop correlations between the mixing flow rates at horizontal cross planes and the lumped sub-volume parameters.

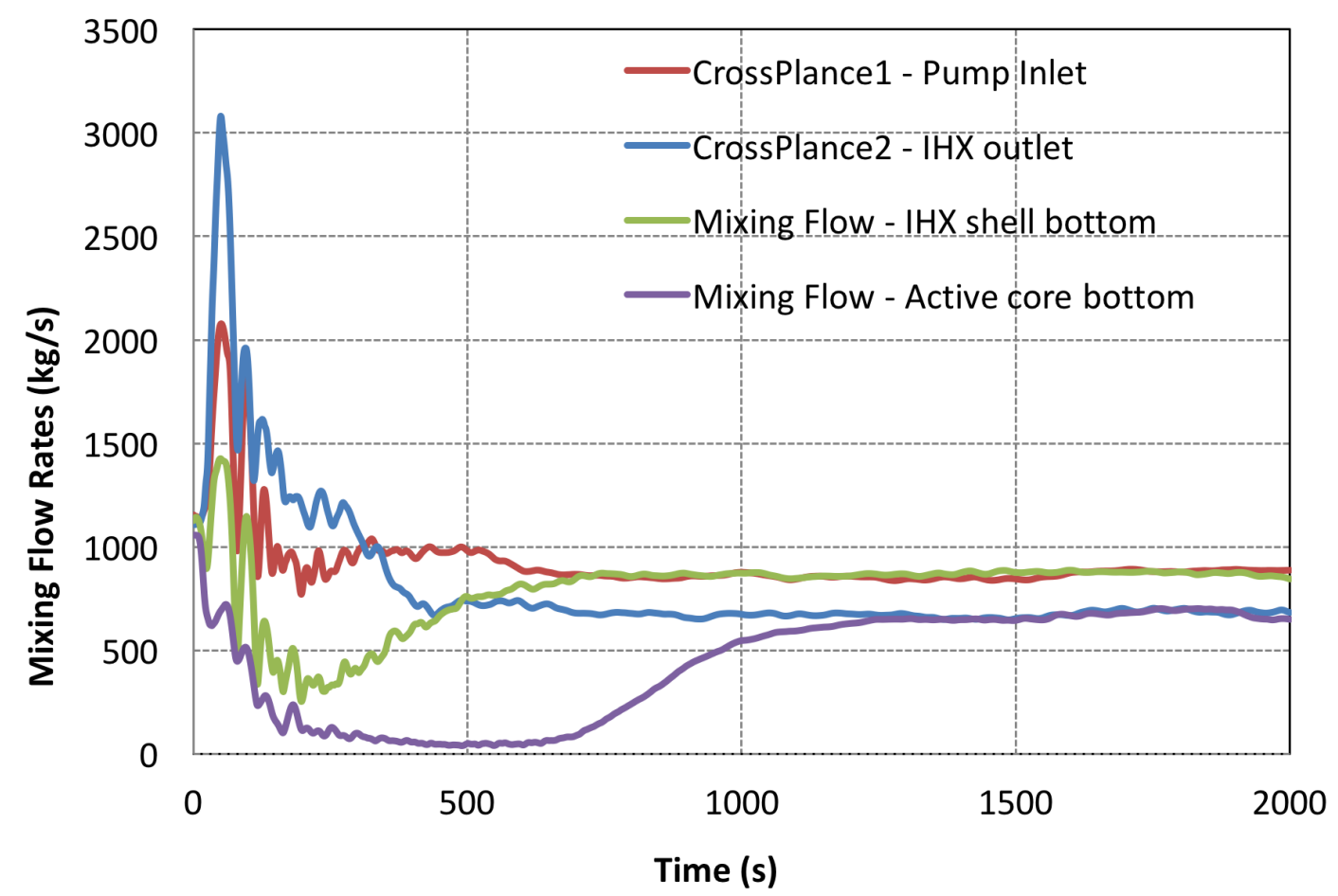

Figure 18. Mixing flow rates at various horizontal cross planes during the BOP-302R test 


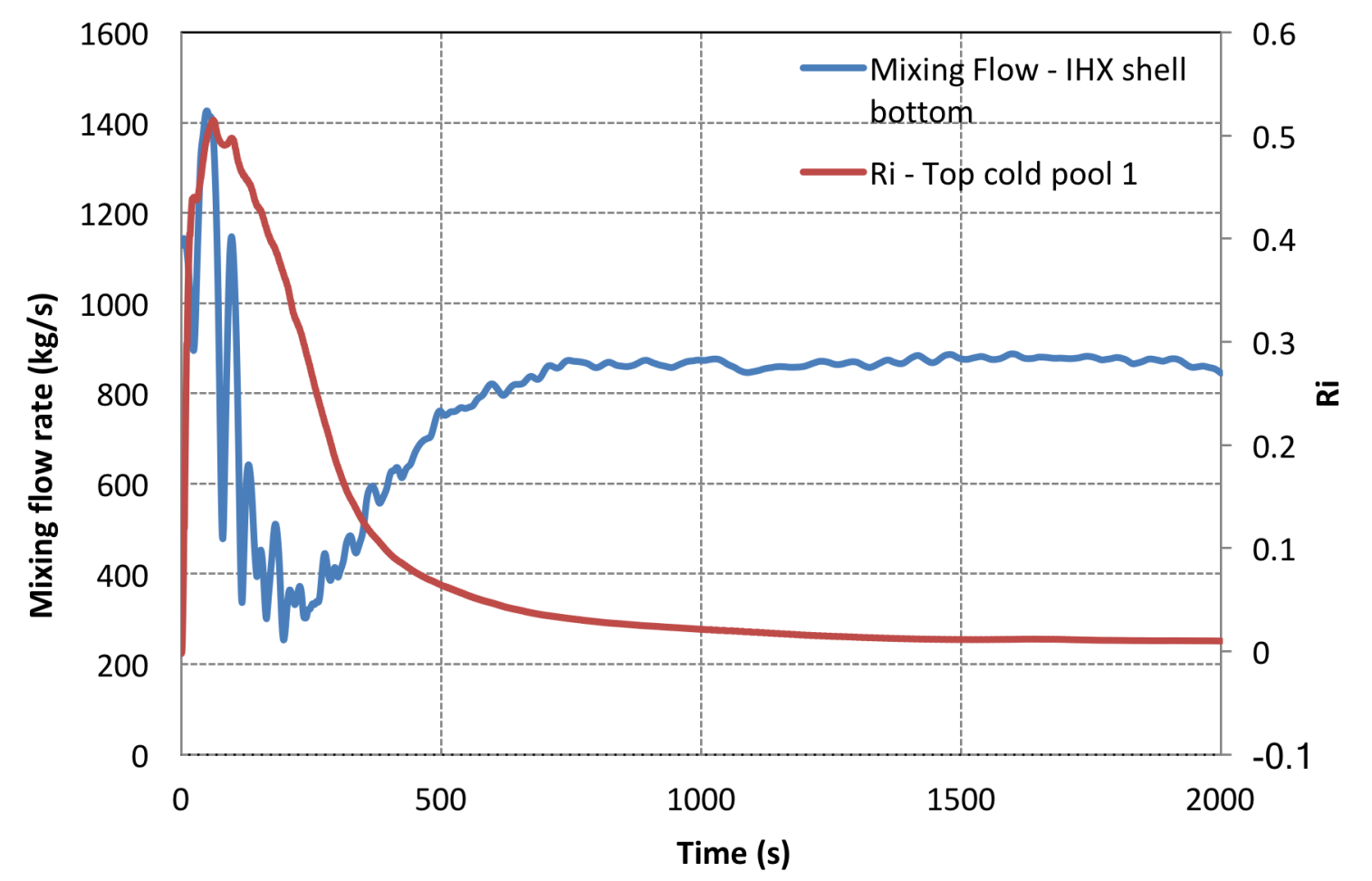

Figure 19. Mixing flow rates and $R i$ at IHX bottom cross plane during the BOP-302R test 


\section{Multi-Dimensional Fluid Model}

While SAM is being developed as a system-level modeling and simulation tool, a threedimensional flow module is also under development. While the 3D module will be useful for useful for a large number of reactor applications, the primary focus so far is to tackle the issue of thermal mixing and stratification modeling in large enclosures of reactor systems.

\subsection{Governing Equations}

The main objective of SAM 3-D fluid model is to provide a computationally efficient modeling capability to model the multi-dimensional flow and thermal stratification phenomena in large enclosures in nuclear systems. To achieve this, the key modeling approaches include:

1) Solving the full 3-D fluid equation;

2) Using only coarse computational meshes;

3) No turbulence modeling;

4) Developing closure models to account for the effects of turbulence and the use of coarse mesh in momentum and energy transport.

The transport equations for three-dimensional single-phase flow in a fluid domain can be described as the following set of partial differential equations. The mass, momentum, and energy equations are closed by the equation of state of the fluid.

$$
\begin{gathered}
\frac{\partial \rho}{\partial t}+\nabla \cdot(\rho \vec{v})=0 \\
\frac{\partial(\rho \vec{v})}{\partial t}+\nabla \cdot(\rho \vec{v} \vec{v})=-\nabla p+\nabla \cdot \overline{\bar{\tau}}+\rho \vec{f} \\
\frac{\partial(\rho h)}{\partial t}+\nabla \cdot(\rho h \vec{v})=-\nabla \cdot \vec{q}^{\prime \prime}+q_{v}^{\prime \prime \prime}+\frac{D p}{D t}+(\overline{\bar{\tau}}: \nabla \vec{v}) \\
\rho=\rho(p, T)
\end{gathered}
$$

Expanding the velocity vector and the momentum equation in three coordinates and simplifying the energy conservation equation, the set of governing equations can be written in the conservative form (Equation 4-2) or in the non-conservative form (Equation 4-3). 


$$
\begin{gathered}
\frac{\partial \rho}{\partial t}+\frac{\partial(\rho u)}{\partial x}+\frac{\partial(\rho v)}{\partial y}+\frac{\partial(\rho w)}{\partial z}=0 \\
\frac{\partial \rho u}{\partial t}+\frac{\partial(\rho u u)}{\partial x}+\frac{\partial(\rho u v)}{\partial y}+\frac{\partial(\rho u w)}{\partial z}=-\frac{\partial p}{\partial x}+\nabla \cdot \vec{\tau}_{x} \\
\frac{\partial \rho v}{\partial t}+\frac{\partial(\rho v u)}{\partial x}+\frac{\partial(\rho v v)}{\partial y}+\frac{\partial(\rho v w)}{\partial z}=-\frac{\partial p}{\partial y}+\nabla \cdot \vec{\tau}_{y} \\
\frac{\partial \rho w}{\partial t}+\frac{\partial(\rho w u)}{\partial x}+\frac{\partial(\rho w v)}{\partial y}+\frac{\partial(\rho w w)}{\partial z}=-\frac{\partial p}{\partial z}+\nabla \cdot \vec{\tau}_{z}+\rho g \\
\frac{\partial(\rho h)}{\partial t}+\frac{\partial(\rho u h)}{\partial x}+\frac{\partial(\rho v h)}{\partial y}+\frac{\partial(\rho w h)}{\partial z}=\nabla\left(k_{e f f} \nabla T\right)+q_{v}^{\prime \prime \prime} \\
\rho=\rho(p, T)
\end{gathered}
$$

In which, $t$ : time; $(x, y, z)$ : the coordinate; $\rho$ : coolant density; $(u, v, w)$ : velocity vector; $g$ : the acceleration due to gravity; $p$ : pressure; $T$ : temperature; $h$ : enthalpy; $\overline{\bar{\tau}}$ : the shear stress and dependent on the velocity gradients and fluid properties, and for Newtonian fluid, $\tau_{i i}=2 \mu \frac{\partial v_{i}}{\partial x_{i}}-$ $\frac{2}{3} \mu \nabla \vec{v}$, and $\tau_{i j}=\tau_{j i}=\mu\left(\frac{\partial v_{i}}{\partial x_{j}}+\frac{\partial v_{j}}{\partial x_{i}}\right) ; k_{e f f}$ : effective thermal conductivity, and $k_{e f f}=k+\alpha$, which accounts for both normal thermal conductivity and additional diffusivity due to turbulence and the use of coarse mesh; $q_{v}^{\prime \prime \prime}$ : volumetric heat source.

$$
\begin{gathered}
\frac{\partial \rho}{\partial t}+\frac{\partial(\rho u)}{\partial x}+\frac{\partial(\rho v)}{\partial y}+\frac{\partial(\rho w)}{\partial z}=0 \\
\rho \frac{\partial u}{\partial t}+\rho u \frac{\partial u}{\partial x}+\rho v \frac{\partial u}{\partial y}+\rho w \frac{\partial u}{\partial z}=-\frac{\partial p}{\partial x}+\nabla \cdot \vec{\tau}_{x} \\
\rho \frac{\partial v}{\partial t}+\rho u \frac{\partial v}{\partial x}+\rho v \frac{\partial v}{\partial y}+\rho w \frac{\partial v}{\partial z}=-\frac{\partial p}{\partial y}+\nabla \cdot \vec{\tau}_{y} \\
\rho \frac{\partial w}{\partial t}+\rho u \frac{\partial w}{\partial x}+\rho v \frac{\partial w}{\partial y}+\rho w \frac{\partial w}{\partial z}=-\frac{\partial p}{\partial z}+\nabla \cdot \vec{\tau}_{z}+\rho g \\
\rho \frac{\partial h}{\partial t}+\rho u \frac{\partial h}{\partial x}+\rho v \frac{\partial h}{\partial y}+\rho w \frac{\partial h}{\partial z}=\nabla\left(k_{e f f} \nabla T\right)+q_{v}^{\prime \prime \prime} \\
\rho=\rho(T)
\end{gathered}
$$

If the incompressible approximation for the shear stress term is used:

$$
\nabla \cdot \vec{\tau}_{x}=\mu \nabla^{2} v_{x}=\mu\left(\frac{\partial^{2} u}{\partial x^{2}}+\frac{\partial^{2} u}{\partial y^{2}}+\frac{\partial^{2} u}{\partial z^{2}}\right)
$$

SAM utilizes the object-oriented application framework MOOSE [21] and its underlying software libraries to leverage the available advanced software environments and numerical methods. High-order discretization schemes in time and space are available. Because of its 
dependence on the MOOSE framework, the SAM 3-D fluid model is also implemented in finite element method (FEM). It is well known that finite element analysis of incompressible flows requires stabilization to avoid potential numerical instabilities. The presence of advection terms (first order terms) in the governing equations can result in spurious node-to-node oscillations [20]. The Streamline-Upwind/Petrov-Galerkin (SUPG) and the Pressure-Stabilizing/PetrovGalerkin (PSPG) scheme are implemented in SAM to resolve the numerical instability issues.

The SUPG method, introduced from Brooks and Hughes in Reference [30], can be considered as the first successful stabilization technique to prevent oscillations in convectiondominated problems in FEM. The main concept is to introduce artificial diffusion in the streamline direction only, interpreted as a modification of the test function of the advection terms, and to enforce consistency, such that this modified test function is applied to all terms of the weak form. The term "artificial diffusion" is not fully applicable any longer because the stabilized weak form cannot be manipulated such that only a diffusion term is extracted. The exact solution of the original problem still satisfies the SUPG stabilized weak form.

Considering a PDE of the general form,

$$
L u=f
$$

where $L$ is any differential operator. The SUPG weak form of the problem is:

$$
\int_{\Omega} w^{*} *(L u-f) d \Omega=0
$$

The standard Bubnov-Galerkin test functions $w$ are modified by a streamline upwind perturbation of the kind:

$$
w^{*}=w+\tau * L_{a d v} w=w+\tau * \nabla w
$$

Where $L_{a d v}$ is the advective part of the whole operator $L$, and $\tau$ is the stabilization parameter that weights the perturbation. Note that the perturbation is multiplied with the residual form of the differential equation. Thereby, consistency is fulfilled from the beginning in that the exact solution also fulfills the stabilized weak form exactly. It is because of this property of the SUPG stabilization (and the PSPG stabilization mentioned later) that numerical oscillations are prevented without introducing excessive numerical diffusion, and therefore without compromising the accuracy of the solution.

The Pressure-Stabilizing/Petrov-Galerkin (PSPG) method is a common technique used for the stabilization of the Stokes equations. The PSPG stabilization term, similar to that of SUPG, consists of a perturbation of the test function multiplied with the residual of the momentum equation, but it is added to the weak form of the continuity equation.

For the incompressible Navier-Stokes equations, both SUPG and PSPG (called herein SUPG/PSPG) stabilization schemes are needed to obtain satisfactory results and to establish the pressure-velocity coupling. In this work, the weak forms of the PSPG/SUPG scheme can be derived as: 


$$
\begin{aligned}
&\left(\frac{\partial \rho}{\partial t}+\nabla \rho \vec{v}, \psi\right)+\left(\rho \frac{\partial \vec{v}}{\partial t}+\rho \vec{v} \nabla \cdot \vec{v}+\nabla p-\nabla \cdot \overline{\bar{\tau}}-\rho \vec{f}, \tau_{P S P G} \nabla \psi\right)=0 \\
&\left(\frac{\partial \rho \vec{v}}{\partial t}+\nabla \rho \vec{v} \vec{v}\right.+\nabla p+-\nabla \cdot \overline{\bar{\tau}}-\rho \vec{f}, \psi) \\
&+\left(\rho \frac{\partial \vec{v}}{\partial t}+\rho \vec{v} \nabla \cdot \vec{v}+\nabla p-\nabla \cdot \overline{\bar{\tau}}-\rho \vec{f}, \tau_{S U P G, m} \vec{v} \cdot \nabla \psi\right)=0 \\
&\left(\frac{\partial(\rho h)}{\partial t}+\nabla \cdot(\rho h \vec{v})-\nabla\left(k_{e f f} \nabla T\right)-q^{\prime \prime \prime}, \psi\right) \\
&+\left(\rho \frac{\partial h}{\partial t}+\rho \vec{v} \nabla h-\nabla\left(k_{e f f} \nabla T\right)-q^{\prime \prime \prime}, \tau_{S U P G, e} \vec{v} \cdot \nabla \psi\right)=0
\end{aligned}
$$

in which $\psi$ is the test function; $\tau_{P S P G}, \tau_{S U P G, m}$, and $\tau_{S U P G, e}$ are the stabilization parameters that weights the perturbations; and $(f, \psi)=\int_{\Omega} \psi \cdot f d \Omega$, is an expression of the volume integral.

Note that the regular residuals of all conservation equations are calculated based on the conservative form (Equation 4-2); while the residuals of the stabilization terms are calculated based on the non-conservative form (Equation 4-3). This formulation not only ensures the conservation laws, but also is easier to be implemented. A review of stabilized finite element formulations for incompressible flow, including the SUPG and PSPG schemes, can be found in Reference [31].

Judicious selection of the stabilization parameters, $\tau_{P S P G}$ and $\tau_{S U P G}$, plays a key role in determining the stability and accuracy of the formulations. Various $\tau$ formulations have been proposed in the literature [32]. The stabilization parameters often involve a measure of the local length scale (i.e. the "element length") and other parameters such as the local Reynolds number and Courant numbers. The UGN-based stabilization parameters defined in Reference [32] are adapted in the SAM 3-D fluid model. Instead of the complex formula of the local length scale $h_{U G N}$ defined in Reference [32], the minimum vertex separation for the element is used. The stabilization parameters can be defined as:

$$
\begin{aligned}
& \tau_{P S P G}=\left[\left(\frac{2}{\Delta t}\right)^{2}+\left(\frac{2 U}{h}\right)^{2}+\left(\frac{4 v}{h^{2}}\right)^{2}\right]^{-\frac{1}{2}} \\
& \tau_{S U P G, m}=\left[\left(\frac{2}{\Delta t}\right)^{2}+\left(\frac{2|\vec{v}|}{h}\right)^{2}+\left(\frac{4 v}{h^{2}}\right)^{2}\right]^{-1 / 2} \\
& \tau_{S U P G, e}=\left[\left(\frac{2}{\Delta t}\right)^{2}+\left(\frac{2|\vec{v}|}{h}\right)^{2}+\left(\frac{4 \alpha}{h^{2}}\right)^{2}\right]^{-1 / 2}
\end{aligned}
$$

Where $h$ is the element length scale; $\vec{v}$ is local velocity; $\Delta t$ is the time step size; $v$ is the kinematic viscosity; $\alpha$ is the thermal diffusivity, $\alpha=\frac{k}{\rho C_{p}}$; and $U$ is a global scaling velocity. If $U$ were not defined in the simulation, the local velocity magnitude would be used.

The methodology presented above is very similar to the 1-D FEM model in SAM [19,20]. The above formulation of the finite element fluid model has been implemented based on FEM, and the resulted equation system is solved in a fully coupled manner (i.e. all the unknowns are solved altogether in a large nonlinear system). The full coupling between different physics is achieved through the Jacobian-Free Newton Krylov (JFNK) method [24]. The JFNK method is a multi-level approach in solving large nonlinear systems, using outer Newton's iterations (nonlinear solver) and inner Krylov subspace methods (linear solver). The concept of "Jacobian- 
free" is proposed since deriving and assembling such large Jacobian matrices could be difficult and expensive. However, in most applications, the Krylov subspace methods require preconditioning to be efficient. Although the JFNK method is used, the entries of an approximate Jacobian matrix are still derived and implemented for the preconditioning (Single Matrix Preconditioner) purpose. The execution speed of the code strongly depends on the number of nonlinear (Newton) and linear (Krylov) iterations.

For computationally efficient modeling of the multi-dimensional flow in a coarse-grid CFD (CGCFD) approach, SAM does not implement any RANS-based turbulence models. Instead, it relies on closure models to close the equation system for the shear stress and the effective diffusivity terms. For laminar flow, the full set of fluid equations is solved, in which the shear stress terms are directly modeled.

$$
\begin{gathered}
\tau_{i i}=2 \mu \frac{\partial v_{i}}{\partial x_{i}}-\frac{2}{3} \mu \nabla \vec{v} \\
\tau_{i j}=\tau_{j i}=\mu\left(\frac{\partial v_{i}}{\partial x_{j}}+\frac{\partial v_{j}}{\partial x_{i}}\right)
\end{gathered}
$$

For turbulent flow, additional closure models will be developed to account for the effects of turbulence and the use of coarse mesh in momentum and energy transport. The porous medium formulation has been implemented as an option in the SAM 3-D module, in which $\nabla \cdot \overline{\bar{\tau}}=$ $\alpha|\vec{v}| \vec{v}+\beta \vec{v}, \alpha$ and $\beta$ are porous resistance coefficients. Additionally, a multi-scale modeling hierarchy will be pursued to develop practical predictive capability at the CGCFD level. Highresolution LES simulations will be leveraged to capture the fine details of the phenomena and to inform the needed closure model development. As the SAM 3-D CGCFD model is still under development, the initial effort has been focused on a flexible framework development of the 3-D module, which allows for easy implementation of closure models once they are available.

\subsection{Demonstration Simulations}

Ongoing verification and validation (V\&V) efforts are being pursued along with the SAM 3D module development. A few classical CFD V\&V test problems has been evaluated to demonstrate the laminar flow modeling abilities of the SAM 3-D module, including lid-driven cavity flow, natural convection inside a cavity, and internal flow in parallel channels.

\subsubsection{Lid-driven cavity flow}

A lid-driven cavity flow problem is presented here involving isothermal, incompressible flow in a two-dimensional square cavity. The geometry is shown in Figure 20, in which all the boundaries of the square are walls. The top wall moves in the $x$-direction at a speed of $1 \mathrm{~m} / \mathrm{s}$ while the other three are stationary. Several cases were tested by varying the fluid viscosity. The velocity distributions at various Reynolds number are shown in Figure 21. With the increase of Reynolds number, the primary vortex center moves downward and rightward. Additionally, 
secondary vortices at cavity corners will become more prominent as the Reynolds number increases.

The SAM predictions were also compared with the experiment results [33] for the lid-driven cavity flow problem at $R e=100$. It is shown in Figure 22 that the normalized velocity in $x$ direction, $V_{x}$, at the center vertical line, and the normalized velocity in in $y$-direction, $V_{y}$, at the center horizontal line, agree very well with the available experimental data.

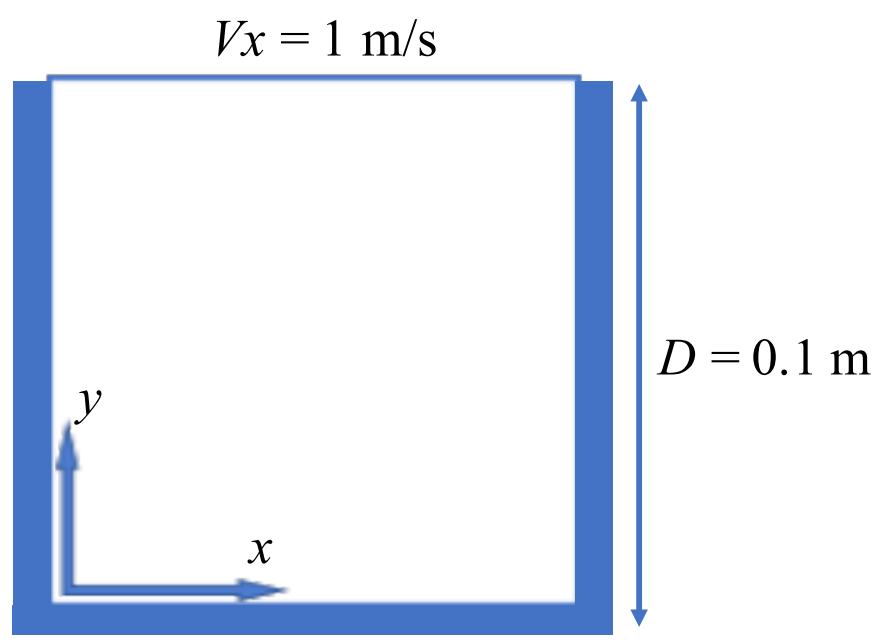

Figure 20: Schematic of the lid-driven cavity flow problem 


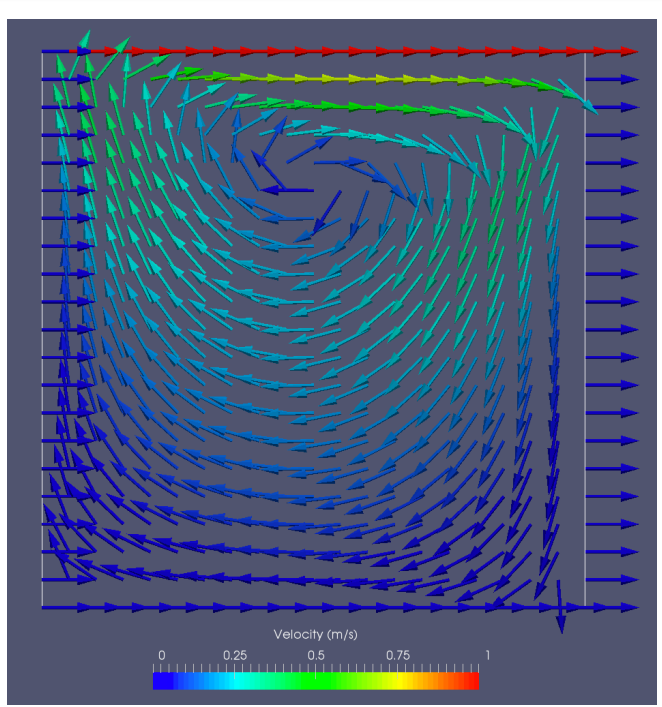

(a) $\operatorname{Re}=10$

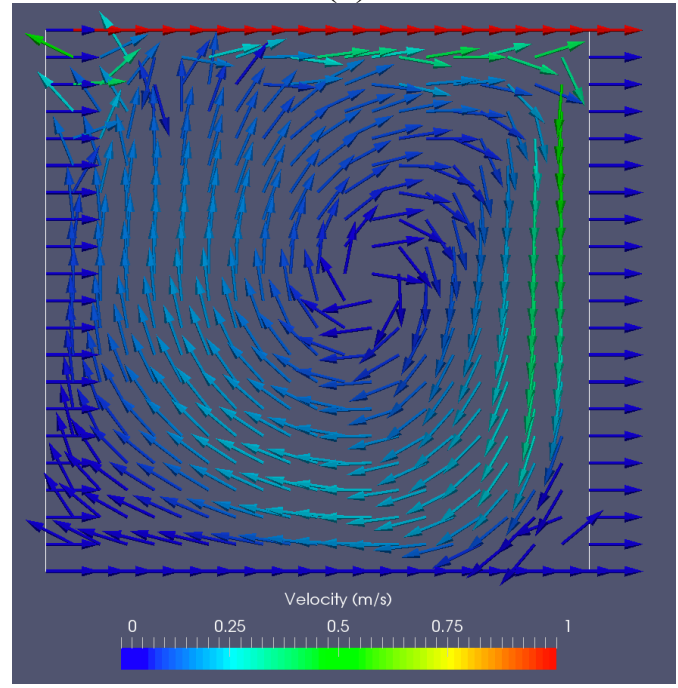

(a) $\mathrm{Re}=1000$

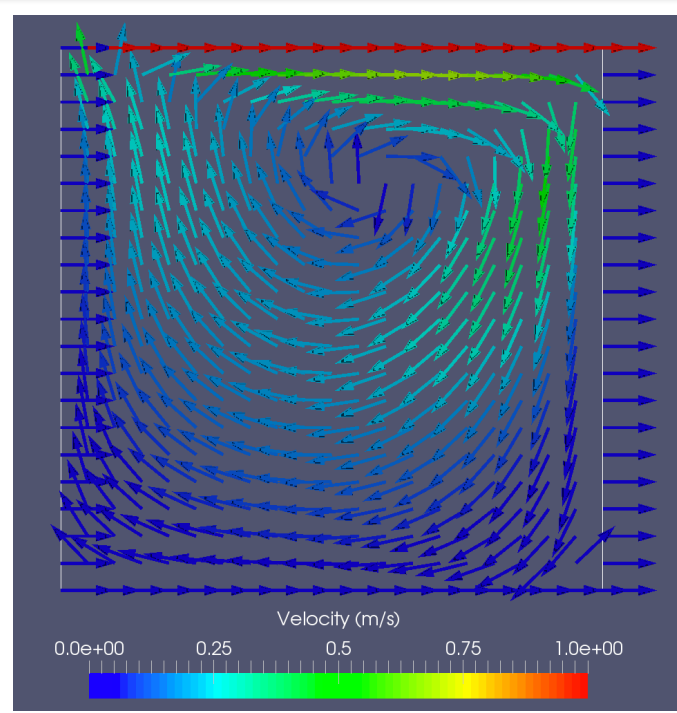

(b) $\mathrm{Re}=100$

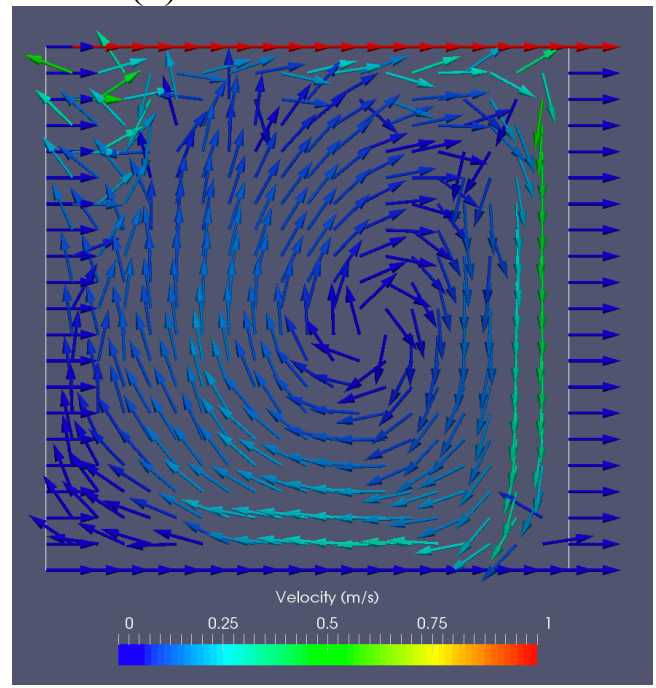

(b) $\mathrm{Re}=2000$

Figure 21: Results of the lid-driven cavity flow problem at various $R e$ 


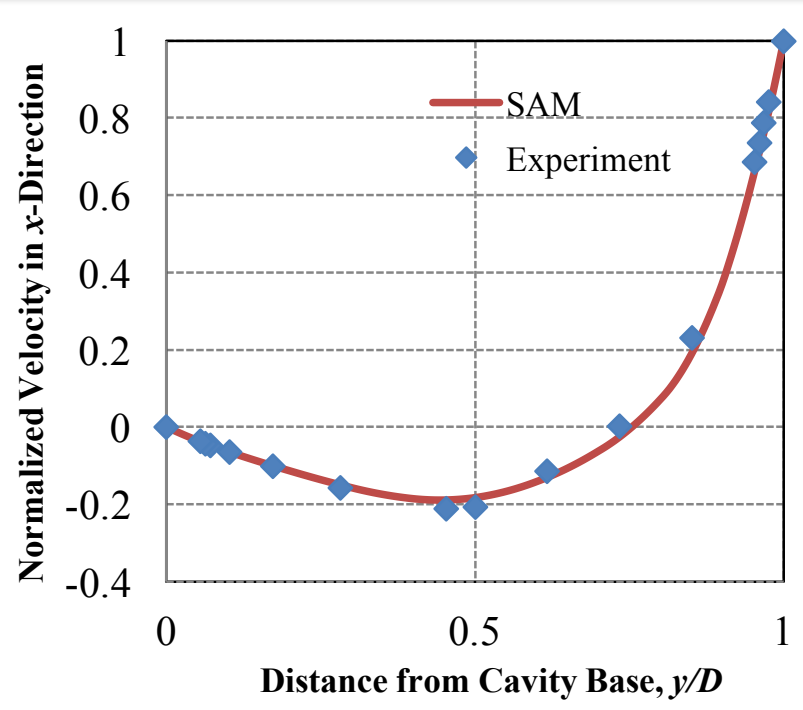

(a) $V_{x}$ at the center vertical line

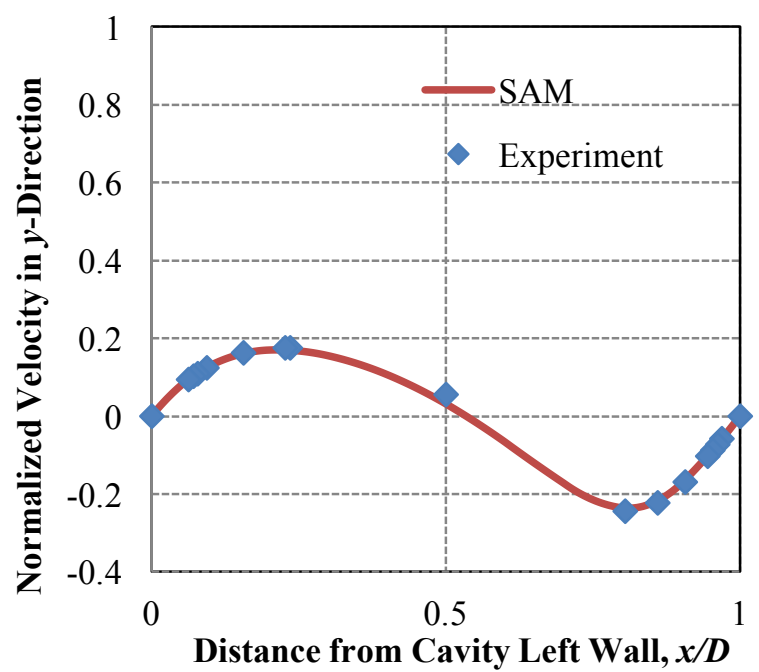

(b) $V_{y}$ at the center horizontal line

Figure 22: Comparisons between SAM and experiment results for the lid-driven cavity flow problem, $R e=100$

\subsubsection{Internal flow between parallel plates}

The SAM 3-D module was also tested for laminar flow in a channel of parallel plates, in which the analytical solutions are available for the fully developed region. The geometry is shown in Figure 23.

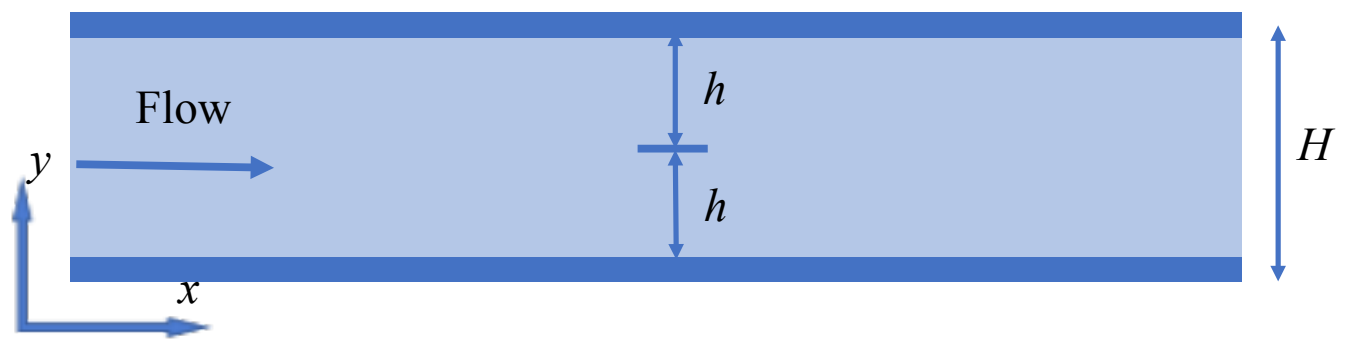

Figure 23: Schematic of flow in a channel of parallel plates

For infinite-wide parallel channels, the equivalent hydraulic diameter can be approximated as: $D_{h} \approx 2 H$. The theoretical results can be derived for incompressible flows. The analytical expression of the entrance length (after which the flow is considered fully developed) can be derived as:

$$
L_{F D}=0.05 R e_{H} H=0.0125 R e_{D_{h}} D_{h}
$$

in which $R e_{H}$ and $R e_{D_{h}}$ are the Reynolds numbers calculated based on the channel height $H$ (distance between parallel plates) and the equivalent hydraulic diameter $D_{h}$.

The velocity distribution and pressure gradient can also be derived for the fully-developed region of this test problem: 


$$
\begin{gathered}
u(y)=\frac{3}{2 h^{2}} u_{\text {avg }}\left(h^{2}-y^{2}\right), h=H / 2 \\
u(0)=\frac{3}{2} u_{\text {avg }} \\
\frac{d p}{d x}=-\frac{3 \mu}{h^{2}} u_{\text {avg }}
\end{gathered}
$$

For the test case with geometry and flow parameters: $H=2 h=0.1 \mathrm{~m} ; L=1 \mathrm{~m}$; $u_{\text {in }}=$ $1 \frac{\mathrm{m}}{\mathrm{s}} ; \rho=100 \frac{\mathrm{kg}}{\mathrm{m}^{3}} ; \mu=0.1 \mathrm{~Pa} \cdot \mathrm{s}$, we can calculate the analytical results. It was confirmed that the following results matched well with the SAM code simulations.

$$
\begin{gathered}
D_{h}=0.2 m, R e_{D_{h}}=200 \\
L_{F D}=0.5 m \\
P(x=0.5)-P(x=1)=\frac{3 \mu}{h^{2}} u_{a v g} L=60 P a
\end{gathered}
$$

The velocity distributions at several distances from the entrances can be found in Figure 24, in which the flow development is clearly observed. The velocity distribution at $x=0.5 \mathrm{~m}$ overlaps with that at $x=1 \mathrm{~m}$, indicating that the flow is fully-developed at $x=0.5 \mathrm{~m}$, which is consistent with the analytical solution.

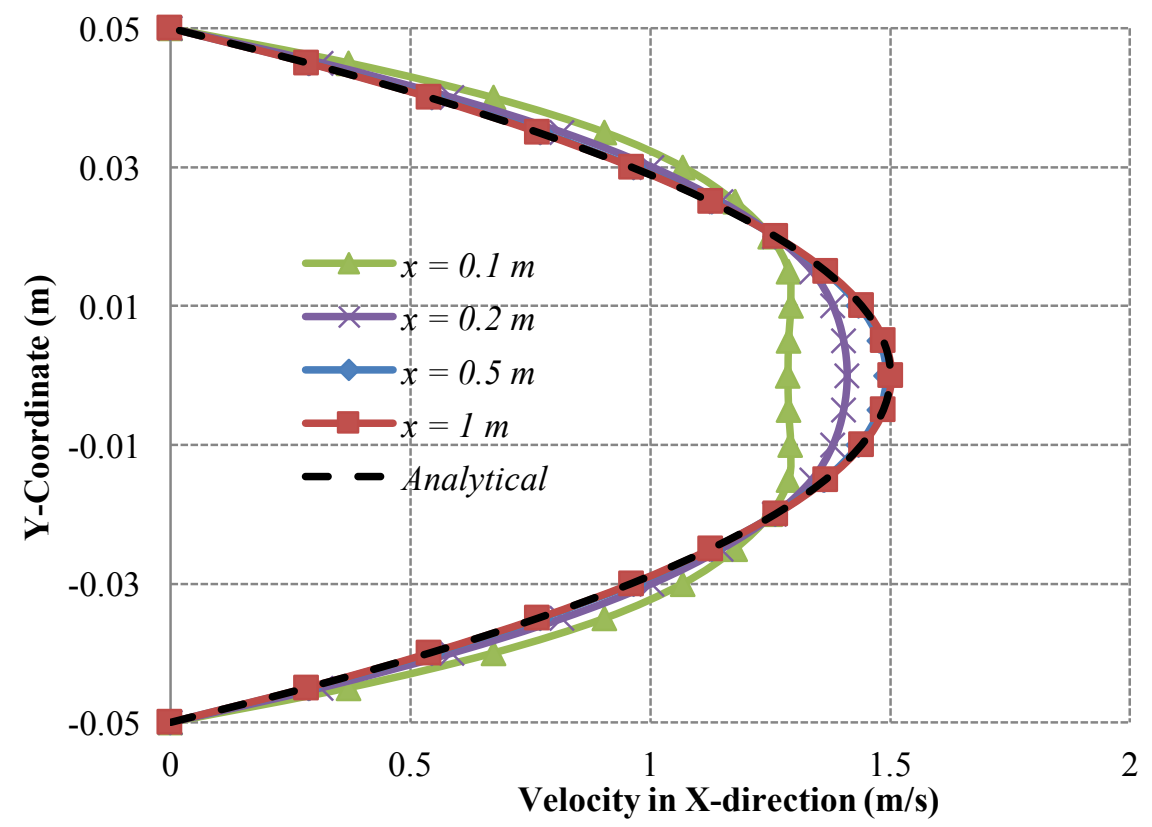

Figure 24: Velocity distributions at different distances from flow inlet 


\subsubsection{Natural circulation flow in a square cavity}

Natural convection flow in enclosures has many thermal engineering applications. Its simplified representative in a square cavity, as shown in Figure 25, has been studied extensively with both experimental and computational studies in the literature. The left wall boundary is fixed constant at a hot temperature while the right wall temperature is fixed constant at a cold temperature. The adiabatic boundary conditions are applied to the top and bottom walls.

Several cases were simulated in SAM with different Rayleigh numbers. The cavity temperature and velocity distributions at various Rayleigh number are shown in Figure 26 and Figure 27. With the increase of Rayleigh number, the flow and thermal boundary layers near the hot and cold walls were both reducing, indicating increased natural circulation flow rates in the cavity. The normalized temperature $\left(T^{*}=\frac{T-T_{\text {cold }}}{T_{\text {hot }}-T_{\text {cold }}}\right)$ distributions from SAM simulations at $y=$ 0.5 are shown in Figure 28 for cases with various Rayleigh numbers. At $R a=10^{3}$, a linear temperature distribution was found from the left to the right walls. With the increase of Rayleigh numbers, strong temperature gradients were observed near the left and right walls, indicating the changes from conduction-dominant to convection-dominant heat transfer.

The SAM simulation results were also compared with the experiment results [34] in the natural convection square cavity at $R a=1.89 \times 10^{5}$. The normalized temperature distributions at the center-horizontal line agreed very well with the available experimental data, as shown in Figure 29.

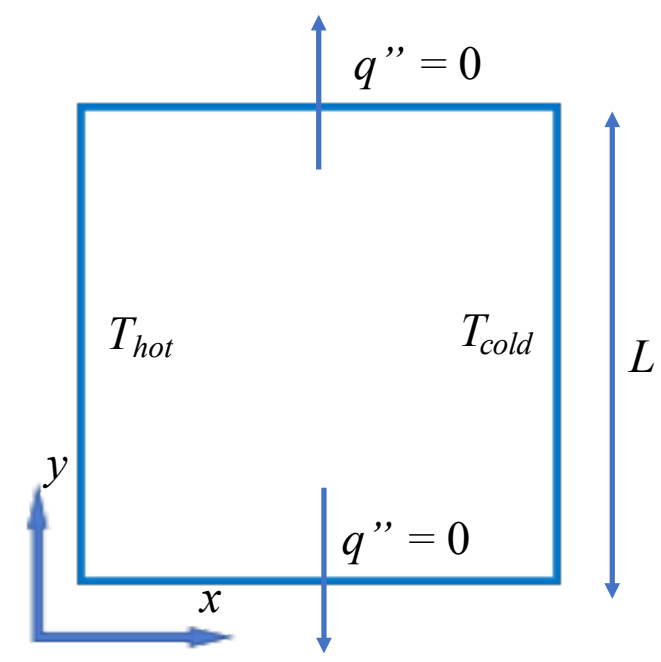

Figure 25: Schematic of natural circulation flow problem in a square cavity 


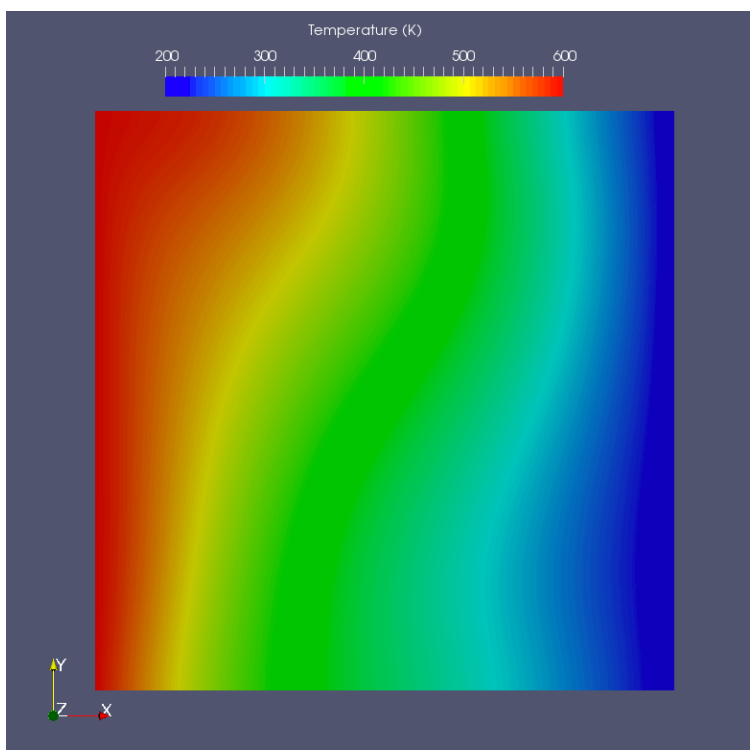

(a) $\mathrm{Ra}=1 \mathrm{e} 3$

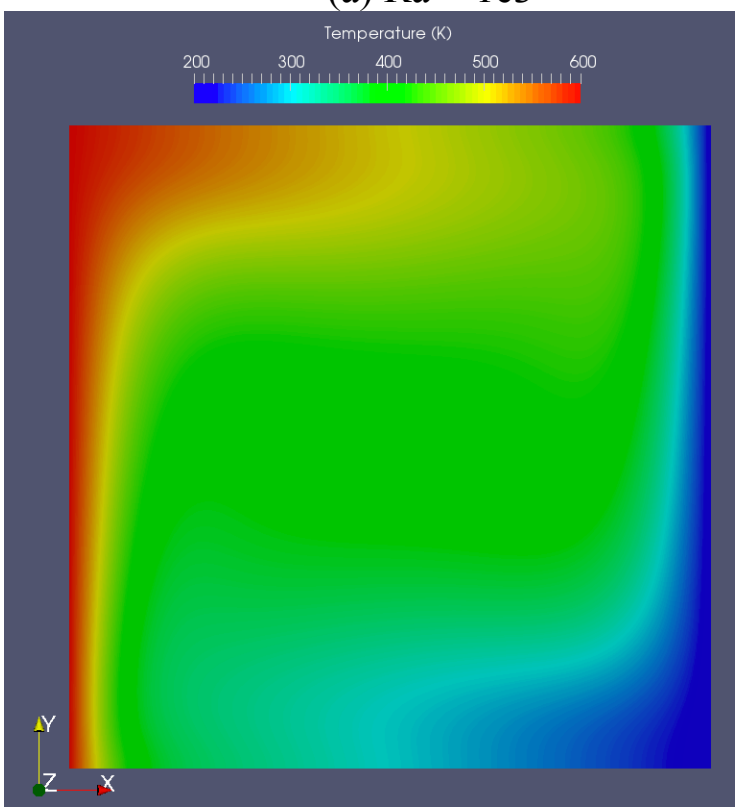

(c) $\mathrm{Ra}=1 \mathrm{e} 5$

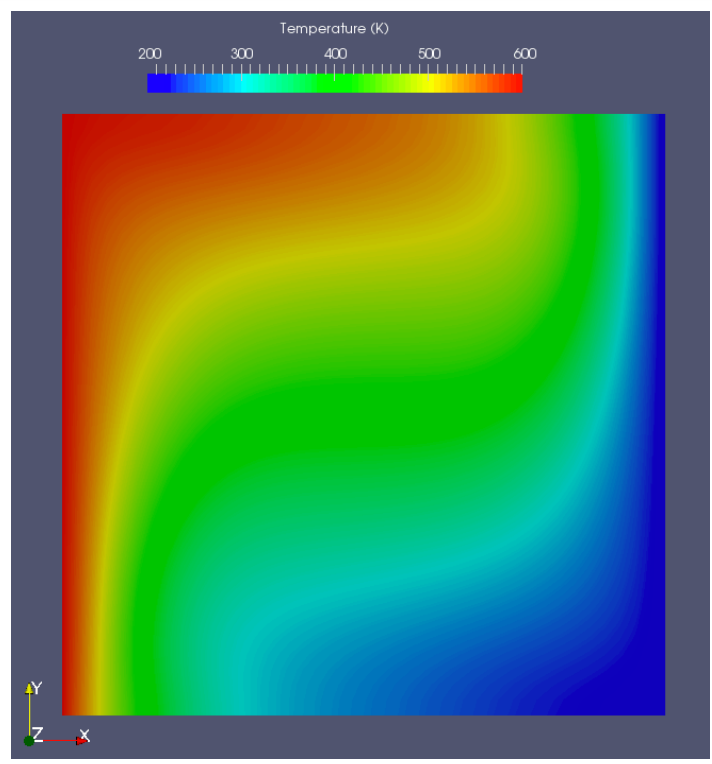

(b) $\mathrm{Ra}=1 \mathrm{e} 4$

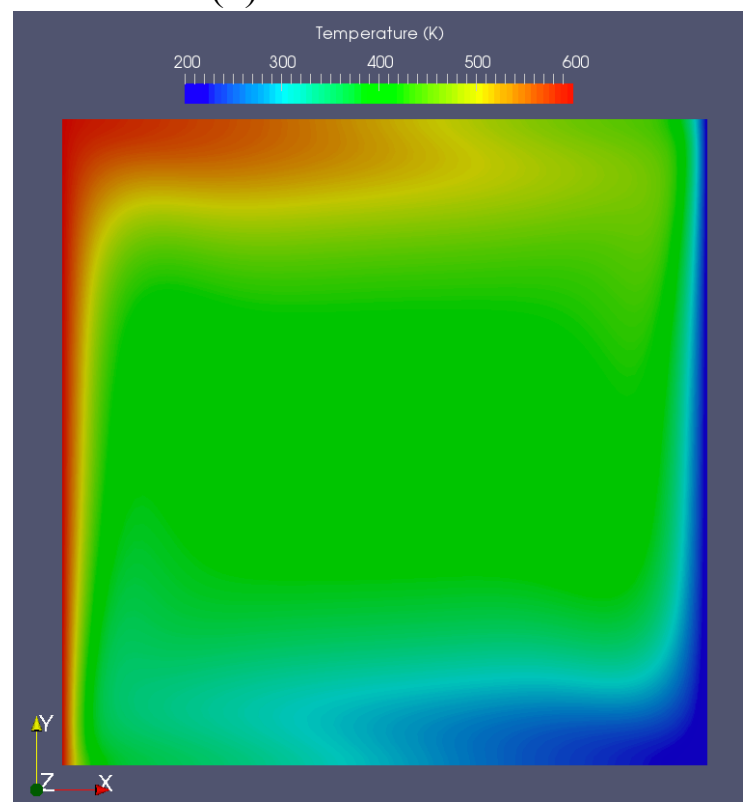

(d) $\mathrm{Ra}=1 \mathrm{e} 6$

Figure 26: Temperature distributions in the square cavity at various $R a$ 


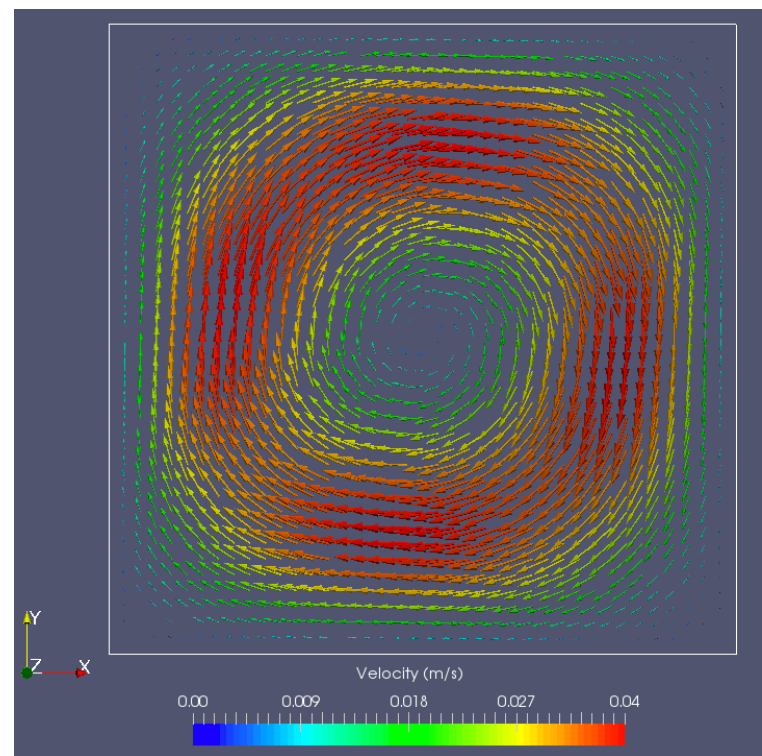

(a) $\mathrm{Ra}=1 \mathrm{e} 3$

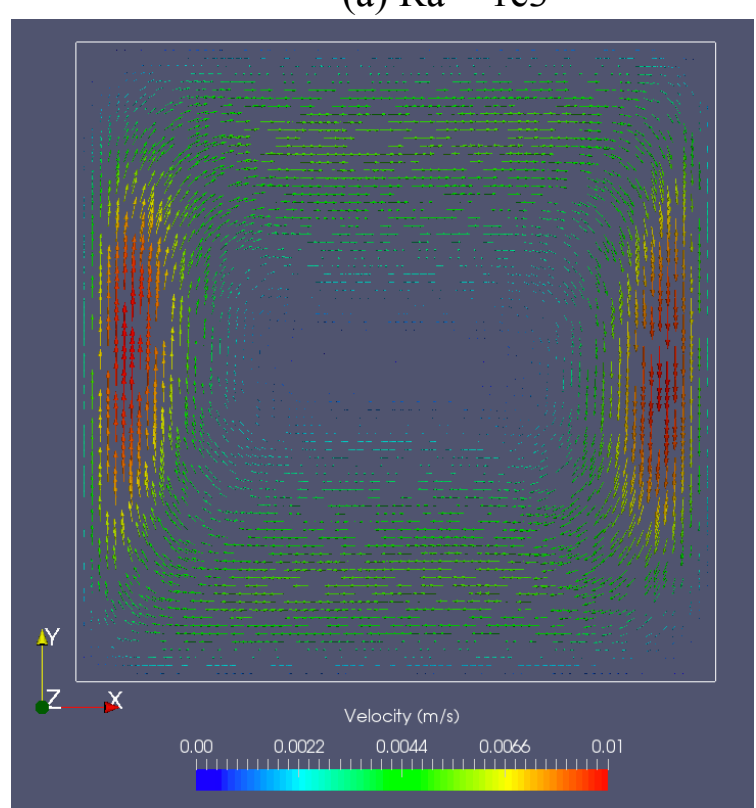

(c) $\mathrm{Ra}=1 \mathrm{e} 5$

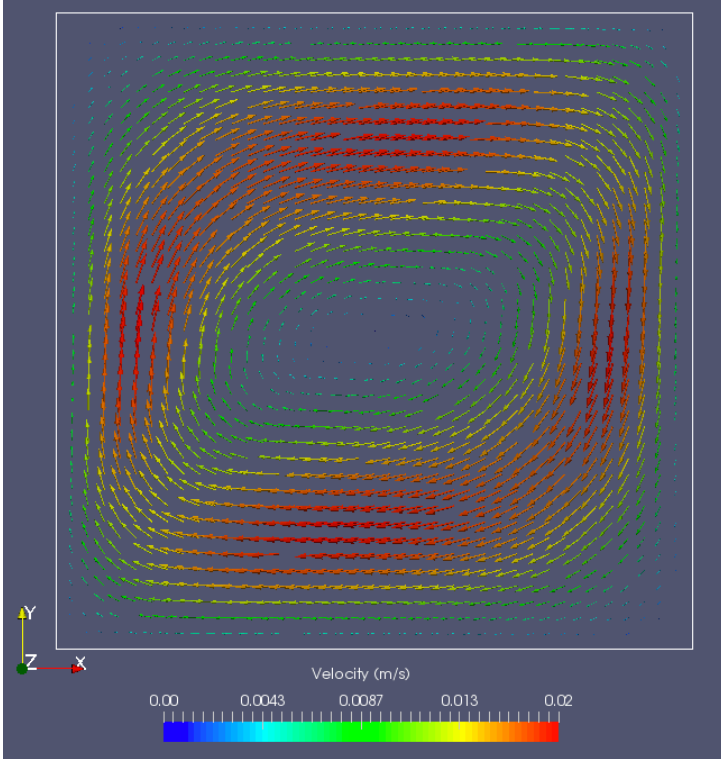

(b) $\mathrm{Ra}=1 \mathrm{e} 4$

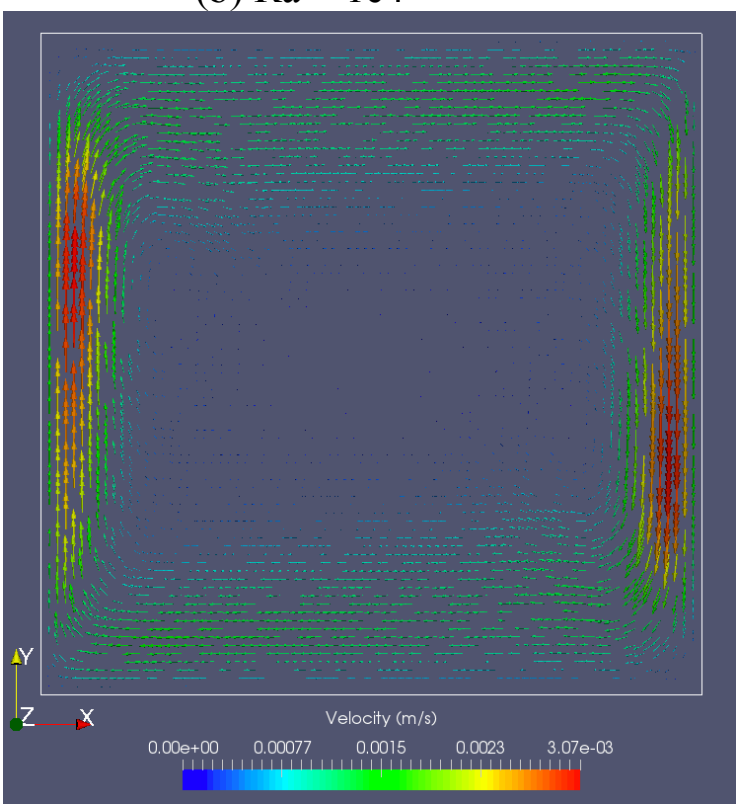

(d) $\mathrm{Ra}=1 \mathrm{e} 6$

Figure 27: Velocity vectors in the square cavity at various $R a$ 


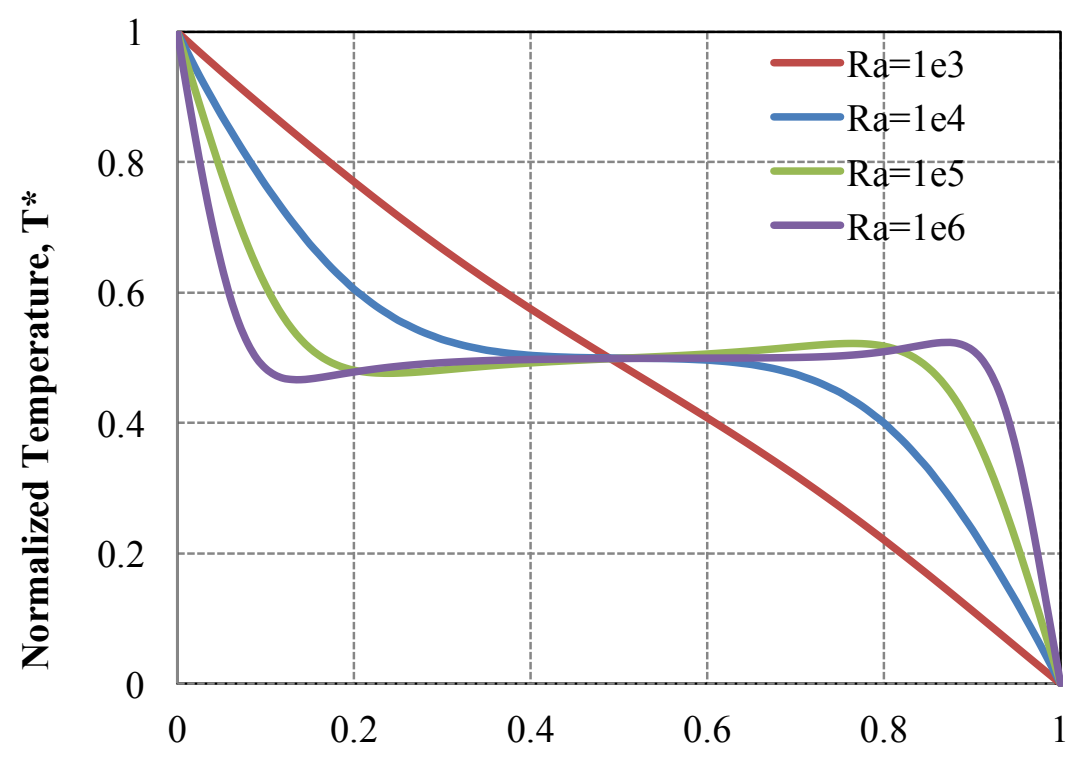

Distance from cavity left wall, $x / L$

Figure 28: Temperature distributions in the square cavity at various $R a, y / L=0.5$

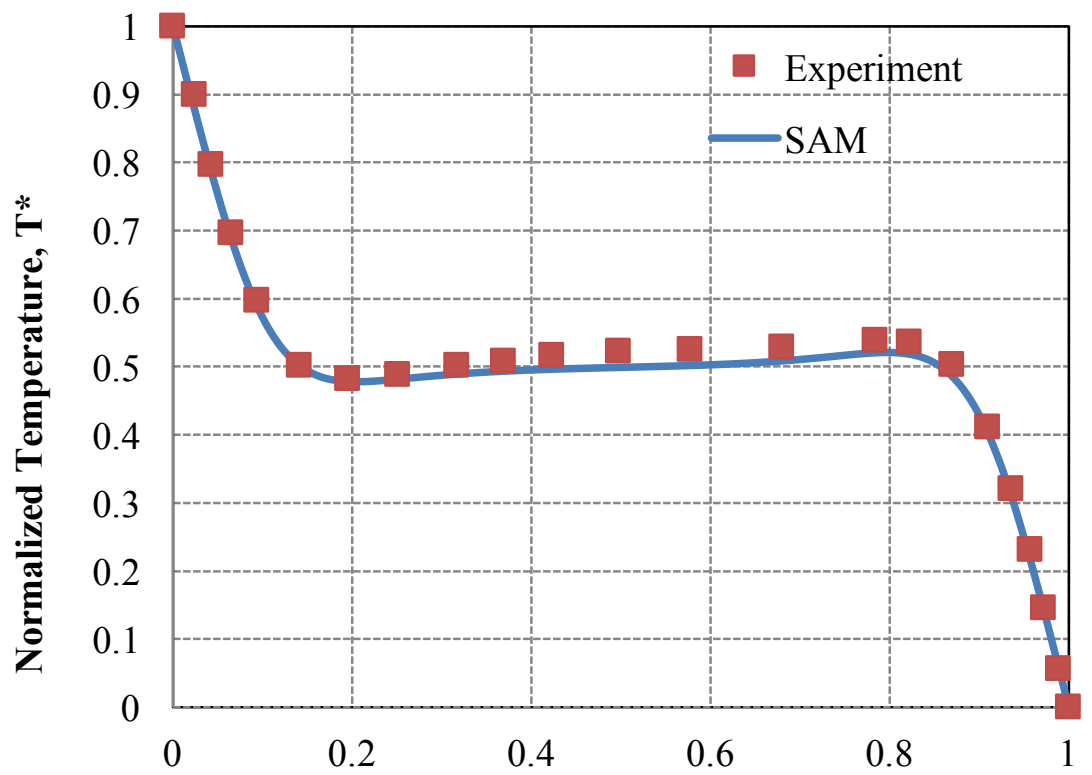

Distance from cavity left wall, $x / L$

Figure 29: Comparison between experiment and SAM predictions, $R a=1.89 \times 10^{5}, y / L=$ 0.5

\subsection{Summary and Path Forward}

While SAM is being developed as a system-level modeling and simulation tool, a reducedorder three-dimensional module is being developed to model multi-dimensional flow and thermal mixing and stratification in large enclosures during transients. An efficient and accurate thermal stratification modeling capability embedded in a system analysis code is very desirable to improve the accuracy of reactor safety analyses and to reduce modeling uncertainties. 
The framework of a 3-D FEM flow model has been developed and implemented in SAM. To prevent the potential numerical instability issues, the SUPG and PSPG formulations have been implemented. Several verification and validation tests were performed, including lid-driven cavity flow, natural convection inside a cavity, and laminar flow in a channel of parallel plates. Based on the comparisons with the analytical solutions and experimental results, it is demonstrated that the developed 3-D fluid model can perform very well for a range of laminar flow problems.

This 3-D flow model is based on solving the primitive variables in the conservative form of the governing equations for incompressible but thermally expandable flows. Combined with the use of the JFNK solution method and high-order discretization schemes, this flow model has great potential for both efficient and accurate multi-dimensional flow simulations. Continued developments will be focused on closure model developments to capture the effects of turbulence and the use of coarse mesh in momentum and energy transport, and on additional code verifications and validations. 


\section{ACKNOWLEDGMENTS}

This work is supported by U.S. DOE Office of Nuclear Energy's Nuclear Energy Advanced Modeling and Simulation (NEAMS) program. We gratefully acknowledge use of the Blues cluster in the Laboratory Computing Resource Center and the Eddy cluster in the Nuclear Engineering Division at Argonne National Laboratory.

\section{REFERENCES}

1. S. Moriya, N. Tanaka, N. Katano and A. Wada, "Effects of Reynolds Number and Richardson Number on Thermal Stratification in Hot Plenum," Nuclear Engineering and Design, Vol. 99, 441-451, (1987).

2. Y. Ieda, I. Maekawa, T. Muramatsu and S. Nakanishi, "Experimental and Analytical Studies of the Thermal Stratification Phenomenon in the Outlet Plenum of Fast Breeder Reactors," Nuclear Engineering and Design, Vol. 120, 403-414, (1990).

3. Auban, et. al., "Investigation of Large-scale Gas Mixing and Stratification Phenomena related to LWR Containment Studies in the PANDA Facility," Nuclear Engineering and Design, Vol. 237, pp. 409-419 (2007).

4. M. Andreani, A. Badillo, and R. Kapulla, "Synthesis of the OECD/NEA-PSI CFD benchmark exercise," Nuclear Engineering and Design 299, 59-80, (2016).

5. H. Zhao and P. Peterson, "An Overview of Modeling Methods for Thermal Mixing and Stratification in Large Enclosures for Reactor Safety Analysis," Proceedings of NUTHOS-8, Shanghai, China, October 10-14, (2010).

6. T.H. Fanning, (Ed.), The SAS4A/SASSYS-1 Safety Analysis Code System, ANL/NE12/4. Argonne National Laboratory, 2012.

7. INL, RELAP5-3D Code Manual Volume I: Code Structure, Systems Models, and Solution Methods, INEEL-EXT-98-00834 Rev. 2.0, (2002).

8. M. Robert, M. Farvacque, M. Parent, and B. Faydide, "CATHARE 2 V2.5: A Fully Validated CATHARE Version for Various Applications," Proceedings of NURETH-10, Seoul, Republic of Korea, October 5-9, 2003.

9. US Nuclear Regulatory Commission, MELCOR Computer Code Manuals, NUREG/CR-6119, Vol. 2, Rev. 3, SAND 2005-5713 (2005).

10. P. F. Peterson, "Scaling and analysis of mixing in large stratified volumes," International Journal of Heat and Mass Transfer, Vol. 37, no. 1, pp. 97-106, 1994.

11. P. F. Peterson and R. Gamble, "Scaling for forced-convection augmentation of heat and mass transfer in large enclosures by injected jets," Transactions of the American Nuclear Society, Vol. 78, pp. 265-266, 1998.

12. H. Zhao, Computation of Mixing in Large Stably Stratified Enclosures, Ph.D. dissertation, University of California, Berkeley (2003).

13. S. Kuhn, H. K. Kang, and P. F. Peterson, "Study of mixing and augmentation of natural convection heat transfer by a forced jet in a large enclosure," Journal of Heat Transfer, Vol. 124, no. 4, pp. 660-666, 2002.

14. H. Zhao, L. Zou, H. Zhang, "Simulation of thermal stratification in BWR suppression pools with one dimensional modeling method," Annals of Nuclear Energy, 63, 533-540, (2014). 
15. A. Kraus and R. Hu, "CFD Analysis of Upper Plenum Flow for a Sodium-Cooled Small Modular Reactor," Proceedings of NURETH-16, Chicago, IL, US, August 30 September 4, 2015.

16. T. H. Fanning and J. W. Thomas, "Integration of CFD into systems analysis codes for modeling thermal stratification during SFR transients," Proceedings of NURETH-14, Toronto, ON, Canada, September 25-30, 2011.

17. R. Hu, J. W. Thomas, and T. H. Fanning, "Strategy for Multi-Scale Single-Phase Flow Coupling," ANL/NE-13/4, Argonne National Laboratory, March 2013.

18. R. Hu, SAM Theory Manual, Argonne National Laboratory, ANL/NE-17/4, March 2017.

19. R. Hu, "A fully-implicit high-order system thermal-hydraulics model for advanced nonLWR safety analyses," Annals of Nuclear Energy, Vol. 101, 174-181, (2017).

20. R. Hu, "An Advanced One-Dimensional Finite Element Model for Incompressible Thermally Expandable Flow", Nuclear Technology, Vol. 190, No. 3, 313-322, (2015).

21. D. Gaston, C. Newman, G. Hansen, and D. Lebrun-Grandi'e, "MOOSE: A parallel computational framework for coupled systems of nonlinear equations," Nuclear Engineering and Design, vol. 239, pp. 1768-1778, (2009).

22. Kirk B. S., Peterson J. W., Stogner R. H., et al., 2006. "libMesh: A C++ Library for Parallel Adaptive Mesh Refinement/Coarsening Simulations," Engineering with Computers, 22(3-4): 237-254.

23. Balay S., Brown J., et al, 2017. PETSc Web page, http://www.mcs.anl.gov/petsc.

24. D. A. Knoll and D. E. Keyes, "Jacobian-free Newton-Krylov Methods: A Survey of Approaches and Applications," Journal of Computational Physics, Vol. 193, pp.357397 (2004).

25. Berry R. A., Peterson J. W., Zhang H., et al., 2015. "RELAP-7 Theory Manual," INL/EXT-14-31366, Idaho National Laboratory.

26. L. L. Briggs, et al., "EBR-II Passive Safety Demonstration Tests Benchmark Analyses Phase 2", Proceedings of NURETH-16, Chicago IL, USA, August 30-September 3, 2015.

27. T. Sumner and A. Moisseytsev, "Simulations of the EBR-II Tests SHRT-17 and SHRT-45R", Proceedings of NURETH-16, Chicago IL, USA, August 30-September 3, 2015.

28. R. Hu and T. Sumner, "Benchmark Simulations of the Thermal-Hydraulic Responses during EBR-II Inherent Safety Tests using SAM”, Proceedings of ICAPP'16, San Francisco CA, April 17-20, 2016.

29. K. Huber and J. Thomas, "Coupled Calculations of SAS4A/SASSYS-1 and STAR-CCM+ for the SHRT-45R Test in EBR-II," Proceedings of NURETH-16, Chicago IL, USA, August 30-September 3, 2015.

30. A.N. Brooks, T.J.R. Hughes, "Streamline upwind/Petrov-Galerkin formulations for convection dominated flows with particular emphasis on the incompressible Navier-Stokes equations," Comp. Methods Appl. Mech. Engrg., 32, 199 - 259, (1982).

31. T.E. Tezduyar, "Stabilized Finite Element Formulations for Incompressible Flow Computations," Advances in Applied Mechanics, Vol. 28, 1-44, (1992).

32. T. Tezduyar and Sunil Sathe, "Stabilization Parameters in SUPG and PSPG Formulations," Journal of Computational and Applied Mechanics, Vol. 4, No. 1, pp. 71-88, (2003). 
33. U. Ghia, K.N. Ghia, and C.T. Shin, "High-Re solutions for incompressible flow using the Navier-Stokes equations and a multigrid method", Journal of Computational Physics, Vol. 48, pp. 387-411, (1982).

34. R. J. Krane and J. Jessee. "Some detailed field measurements for a natural convection flow in a vertical square enclosure." 1st ASME-JSME thermal engineering joint conference. Vol. 1. ASME New York, 1983. 
Progress Report on SAM Reduced-Order Model Development

September 2017 


\section{Argonne}

Nuclear Engineering Division

Argonne National Laboratory

9700 South Cass Avenue, Bldg. 208

Argonne, IL 60439

www.anl.gov 